\title{
Prioritization of autoimmune disease-associated genetic variants that perturb regulatory element activity in $T$ cells
}

Kousuke Mouri ${ }^{1,9}$, Michael H. Guo ${ }^{2,3,9}$, Carl G. de Boer ${ }^{3,4}$, Gregory A. Newby ${ }^{3,5,6}$, Matteo

Gentili³, David R. Liu ${ }^{3,5,6}$, Nir Hacohen ${ }^{3,7,10, *}$, Ryan Tewhey ${ }^{1,10, *}$, John P. Ray,8,10,*

${ }^{1}$ The Jackson Laboratory, Bar Harbor, ME 04609

${ }^{2}$ Department of Neurology, Perelman School of Medicine, University of Pennsylvania, Philadelphia, PA 19104

${ }^{3}$ Broad Institute of Harvard and MIT, Cambridge, MA 02142

${ }^{4}$ School of Biomedical Engineering, University of British Columbia, Vancouver, BC, Canada V6T 1 Z3

${ }^{5}$ Department of Chemistry and Chemical Biology, Harvard University, Cambridge, MA 02138

${ }^{6}$ Howard Hughes Medical Institute, Harvard University, Cambridge, MA 02138

${ }^{7}$ Center for Cancer Research, Massachusetts General Hospital, Boston, MA, 02114

${ }^{8}$ Systems Immunology, Benaroya Research Institute, Seattle, WA 98101

${ }^{9}$ These authors contributed equally

${ }^{10}$ Corresponding authors

*Correspondence: nhacohen@mgh.harvarrd.edu (N.H), ryan.tewhey@jax.org (R.T.), jray@,benaroyaresearch.org (J.P.R) 


\begin{abstract}
Genome-wide association studies have uncovered hundreds of autoimmune disease-associated loci; however, the causal genetic variant(s) within each locus are mostly unknown. Here, we perform high-throughput allele-specific reporter assays to prioritize disease-associated variants for five autoimmune diseases. By examining variants that both promote allele-specific reporter expression and are located in accessible chromatin, we identify 60 putatively causal variants that enrich for statistically fine-mapped variants by up to 57.8-fold. We introduced the risk allele of a prioritized variant (rs72928038) into a human T cell line and deleted the orthologous sequence in mice, both resulting in reduced $B A C H 2$ expression. Naïve CD8 T cells from mice containing the deletion had reduced expression of genes that suppress activation and maintain stemness. Our results represent an example of an effective approach for prioritizing variants and studying their physiologically relevant effects.
\end{abstract}




\section{INTRODUCTION}

Genome-wide association studies (GWAS) are a powerful approach for identifying genetic susceptibility loci for autoimmune diseases. However, our ability to draw direct mechanistic insights from GWAS loci has been hampered by challenges in identifying which variant(s) actually cause disease risk at any given locus. Pinpointing the specific causal variant provides insight into the context and mechanism by which the disease association modulates disease risk. There are three major challenges to identifying causal variant(s): 1) at most loci, there are many disease-associated variants due to linkage disequilibrium (LD) between causal and non-causal variants, 2) $\sim 90 \%$ of causal variants reside in non-coding regions ${ }^{1,2}$, where their mechanisms of action are difficult to infer, and 3) the context (e.g., cell-type, cell-state, etc.) in which variants act may at times be difficult to discern, particularly for non-coding variants. Thus, to discern causal variants, we must refine strategies to prioritize and test variants for how they perturb genomic functions, particularly in disease-relevant cell types and states.

Recent methodologies have been developed to distinguish causal variants from those that are non-causal, including inferring the cell types in which they act. Statistical fine-mapping methods can generate credible sets of likely causal variants, with high powered studies able to pinpoint singular causal variants for many disease associations ${ }^{3}$; however, most disease association studies lack sufficient power to definitively distinguish the causal variant(s) for each locus. Experimental and computational methodologies have also been developed to discern putatively causal variants and infer the context in which they act. For instance, overlaying maps of accessible and active chromatin regions through DNase Hypersensitivity I and H3K27ac ChIP-sequencing in many cell types and environmental conditions have enriched for likely causal variants, and these methods can aid in identifying disease-relevant cell types ${ }^{1,2,4,5}$. In 
addition, perturbational studies-- such as massively parallel reporter assays (MPRA) that test genetic variants for their ability to modulate gene expression and CRISPR inhibition which perturbs putative regulatory elements to determine their effect on gene expression-- also enrich for disease-associated variants ${ }^{6,7}$. While each of these methodologies is useful for prioritizing potential causal variants, they all have imperfect accuracy due to differences in how variants act in the context of the assay as compared to disease-relevant states ${ }^{6}$. Thus, once variants are prioritized, they require further mechanistic dissection to determine whether they are causal for disease associations, such as through editing the variants into the genomes of disease-relevant cells.

Here, we used a highly efficacious prioritization scheme on $\sim 18,000$ variants associated with five autoimmune diseases including type 1 diabetes (T1D), inflammatory bowel disease ([IBD], including ulcerative colitis [UC] and Crohn's disease [CD]), rheumatoid arthritis (RA), psoriasis, and multiple sclerosis (MS) to identify likely causal variants. Through integrating MPRA and chromatin accessibility data, we found 60 likely causal variants that enriched up to 57.8-fold for causal variants according to fine-mapping. We further characterized the effects of a single variant (rs 72928038) associated with multiple autoimmune diseases through analyzing the presence of the risk allele in accessible chromatin, using a base editing approach to insert the variant into a human $\mathrm{T}$ cell line, and by constructing mice containing a deletion at the orthologous genomic region. Human T cells heterozygous for the variant have substantial reductions in accessible chromatin containing the risk allele, and insertion of the variant into Jurkat $\mathrm{T}$ cells reduced expression of $B A C H 2$, a transcriptional repressor that negatively regulates effector $\mathrm{T}$ cell differentiation ${ }^{8}$, and positively regulates regulatory $\mathrm{T}$ cell differentiation ${ }^{9}$ and $\mathrm{T}$ cell stemness ${ }^{10}$. Because the region containing rs 72928038 is highly conserved, and the 
orthologous region in mouse is also in a putative cis-regulatory element in mouse $\mathrm{T}$ cells, we created mice with a small deletion in the non-coding region overlapping rs 72928038 to determine its effect on Bach2 and global expression of naïve T cells. We found rs72928038deleted mice to have naïve CD8 T cells with reduced Bach2 expression and reduced expression of naïve $\mathrm{T}$ cell stemness-associated genes, indicating that rs72928038 plays an important role in suppressing naïve $\mathrm{T}$ cell activation. This work demonstrates a framework for combining chromatin accessibility and MPRA to identify variants that impact risk for autoimmune disease and provides a clear example of how to move from variant prioritization to causal effects on cellular outcome in an organismic model.

\section{RESULTS}

Prioritizing autoimmune GWAS variants with MPRA. Because autoimmune diseaseassociated genetic variants are highly enriched in $\mathrm{T}$ cell cis-regulatory elements ${ }^{1,2,5,6,11,12}$, we hypothesized that many disease-causal variants likely alter the activity of $\mathrm{T}$ cell cis-regulatory elements. One way to test the effect of variants on regulatory activities is through testing variant alleles for their differential effects on reporter expression in MPRA ${ }^{13-16}$. To this end, we created MPRA libraries for variants associated with diseases in which $\mathrm{T}$ cells are known to play a role (henceforth collectively referred to as T-GWAS). These diseases include IBD (including CD and $\mathrm{UC})^{17}, \mathrm{MS}^{18,19}, \mathrm{~T}^{20} \mathrm{D}^{20}$ psoriasis $^{21}$, and $\mathrm{RA}^{22}$.

We collected 578 GWAS index variants (representing 531 distinct GWAS loci) and variants in tight $\mathrm{LD}\left(\mathrm{r}^{2}>0.8\right)$ from the above-cited studies, totaling 18,312 variants (Supplementary Table 1). To generate our MPRA, library alleles were synthesized as 200 bp elements centered within their genomic context. We also included 91 positive enhancer controls 
and 506 negative controls used in a previous MPRA study (Supplementary Table 2) ${ }^{13}$. We barcoded the enhancer elements $(\sim 1,000$ barcodes/element $)$ and cloned them into the MPRA vector, followed by inserting a minimal promoter and GFP (Fig. 1a, Supplementary Fig.1a, Methods). After nucleofection of the library into Jurkat T cells, followed by RNA-sequencing of barcodes after $24 \mathrm{~h}$, we found that barcode prevalence in plasmid and cDNA replicates was tightly correlated, and that some barcodes were more present in cDNA than in plasmid libraries, indicative of their higher expression (Supplementary Fig.1b; Supplementary Table 3). We found 7,095 elements that had higher reporter expression than expected from their prevalence in plasmid libraries for at least one variant allele (termed putative cis-regulatory elements, pCREs; Supplementary Fig.1c; Supplementary Table 4); positive enhancer controls generally were pCREs, while negative controls had minimal expression (Supplementary Fig.1d). Of the 7,095 pCRE elements, we found 313 variants that had statistically significant differences in expression between the reference and alternate alleles, which we term expression-modulating variants (emVars) (Fig. 1b; Supplementary Table 4).

We next assessed whether there are specific cis-regulatory phenotypes in which emVars were preferentially found. We observed emVars were most highly enriched in transcription start site (TSS) regions and distal enhancers (Supplementary Fig. 2a and b), with particularly high enrichment at regions marked by $\mathrm{H} 3 \mathrm{~K} 4 \mathrm{me} 3, \mathrm{CAGE}$ and DNase hypersensitivity sites (DHS) (Supplementary Fig. 2c), consistent with many emVars altering regulatory element activity. emVars were also more likely to have allelic bias in ATAC-seq data from hematopoietic cell types and to be a chromatin accessibility quantitative trait locus (caQTL) as compared to MPRA variants with no activity, and emVar allelic effects were correlated with allelic bias and QTL directionality from these data (Supplementary Fig. 3). Consistent with emVars disrupting 
regulatory element activity and chromatin accessibility, we found that their allelic effects were correlated with computationally predicted allelic effects ("delta SVM") in CD4 T cell enhancer elements, with most emVars showing directional concordance with the delta SVM score (Supplementary Fig. 4a). emVars were also much more likely to perturb a transcription factor (TF) motif (according to position weight matrices) when compared to all variants tested in the MPRA assay, with predicted TF binding also correlating strongly with the observed MPRA allelic bias for emVars (Supplementary Fig. 4b-d; Supplementary Tables 4-6). Therefore, TGWAS emVars enrich in regulatory regions and for variants that have orthogonal regulatory phenotypes and allele-specific activities.

Since emVars enrich for variants that impact regulatory activity, we predicted that MPRA could be used to identify causal variants at GWAS loci. Most GWAS loci are thought to have one or a small number of causal variants, with remaining variants statistically associated with a given disease solely due to tight LD with the true causal variant(s). Consistent with this notion, of the 181 GWAS loci for which we found an emVar (31\% of all assessed GWAS loci; Supplementary Fig. 5a), 120/181 loci had only one emVar, and 169/181 loci had four or fewer emVars (Supplementary Fig. 5b).

To test if emVars are identifying causal variants, we next tested whether emVars are enriched for variants identified by statistical fine-mapping. We performed fine-mapping using PICS $^{1}$ for all five autoimmune diseases (Supplementary Tables 7 and 8). Among the various statistical fine-mapping approaches available, we chose to use PICS as it does not require full GWAS summary statistics, which were unavailable for many of the diseases we analyzed. We tested whether emVars are enriched for high posterior probability variants at various posterior probability thresholds. When taking into account all GWAS loci, regardless of whether an 
emVar was identified, emVars enriched up to 3.49-fold for causal variants according to PICS (Supplementary Fig. 6a; Supplementary Table 9). With an understanding that MPRA will not identify variants in all loci such as those where a coding variant is causal, we decided to test enrichments for loci where at least one emVar was identified. Within loci where we identified at least one emVar, we found that emVars were as much as 28.5-fold enriched for causal variants according to PICS (Fig. 1c; Supplementary Table 10). Among loci containing both an emVar and a high posterior probability fine-mapped SNP (posterior inclusion probability [PIP] $>0.5$ ), 45\% of the high PIP fine-mapped SNPs were also emVars. Since the T1D GWAS we used to create our MPRA library also contained statistical fine-mapping data ${ }^{20}$, we assessed the enrichment of emVars for statistically fine-mapped variants from this separate dataset, detecting up to a 4.17fold enrichment (Supplementary Fig. 6b). These data suggest that MPRA is a highly robust approach for prioritizing causal disease variants.

To estimate the sensitivity and specificity of the MPRA, we again leveraged PICS statistical fine-mapping. We constructed credible sets of statistically fine-mapped variants (for example, an $80 \%$ credible set will contain the causal variant $80 \%$ of the time (Supplementary Table 7; see Methods)), similar to the approach utilized in a recent MPRA study ${ }^{14}$. At various PICS credible sets, we calculated the sensitivity of the MPRA to be $18.4 \%$ to $19.7 . \%$, and specificity ranging from $90.8 \%$ to $95.0 \%$. Thus, MPRA can prioritize causal variants at a fifth of all loci while maintaining high specificity.

\section{emVars in $\mathbf{T}$ cell accessible chromatin occur near genes that regulate $\mathbf{T}$ cell function. We} found that active elements within our MPRA were enriched for regions of accessible chromatin from $\mathrm{T}$ cells and other hematopoietic cell types compared to non-hematopoietic cell types (Fig. 
2a), suggesting that MPRA regulatory activity accurately reflects the transcriptional regulation of the cell type in which it is tested. However, because MPRA evaluates the regulatory activity of a sequence outside its genomic context, effects of variants measured by MPRA may differ from the true endogenous effects. We previously performed MPRA on all common variants near TNFAIP3 and observed a higher enrichment for putatively disease-causal variants when taking the intersection of MPRA results and regions of accessible chromatin ${ }^{6}$. To assess whether these prior findings extend to our genome-wide T-GWAS MPRA experiment, we compared all variants tested in MPRA to those that were in DHS regions in T cells. Of the 313 emVars, 60 overlapped a T cell DHS peak (Supplementary Table 4). For genetic associations that had at least one emVar in accessible chromatin, we found up to 57.8-fold enrichment for causal variants according to PICS (Fig. 2b and Supplementary Table 10; when taking into account all GWAS loci tested, there was a 9.3-fold enrichment for causal variants according to PICS, Supplementary Fig. 7 and Supplementary Table 9). We calculated sensitivity and specificity for emVars within PICS credible sets at loci where any variant on the haplotype overlapped a T cell DHS peak. When subsetting loci for those with a variant in DHS, MPRA achieved a sensitivity ranging from $23.1 \%$ to $25.5 \%$ and specificity ranging from $81.1 \%$ to $91.7 \%$. Therefore, emVars that are present in the accessible chromatin of T cells enriched strongly for causal variants, and to a much greater extent than either methodology alone.

Many emVars in accessible chromatin were near (and in most cases were eQTLs for) genes with important roles in $\mathrm{T}$ cell biology, including genes that regulate $\mathrm{T}$ cell differentiation (BACH2, EOMES, RORC, CEBPB), signal transduction (CD28, CTLA4, ICOS, STAT1, STAT2, STAT4, IRF5, NFKB1, NFKB2, RELA, SOCS1), cytokine production (IL2, IL21, IL23), and migration (CCRO) (Fig. 2c). rs654690, associated with psoriasis, IBD, and RA, falls 
preferentially in the accessible chromatin of Tregs and contacts the TAGAP promoter $\sim 50 \mathrm{~kb}$ downstream ${ }^{23}$; TAGAP is a gene that has been shown to play a role in Th17 cell differentiation and thymocyte trafficking ${ }^{24-26}$ (Fig. 3a). The rs654690 disease-risk allele depends on the disease: the RA risk-increasing allele $(\mathrm{C})$ decreases MPRA activity, while the psoriasis and IBD riskincreasing allele (T) increases MPRA activity (Fig. 2c). Disease-risk alleles for two variants, rs 142738614, associated with MS, RA, and UC, and rs3807306, associated with RA, were in moderate LD to each other $\left(r^{2}=0.7\right)$, and in separate regulatory elements of $I R F 5$, a gene with many roles in immunity, including $\mathrm{T}$ cell-intrinsic roles that modulate signaling, migration, and differentiation $^{27}$ (Supplementary Fig. 8a). The risk alleles for both variants drove an increase in reporter expression (Fig. 2c). rs55728265, associated with CD and T1D, is in the 5' UTR of RASGRP1, a gene that regulates $\mathrm{T}$ cell signaling and differentiation ${ }^{28,29}$ (Supplementary Fig. 8b); the rs55728265 risk allele increases reporter expression (Fig. 2c). rs72928038, associated with $\mathrm{T} 1 \mathrm{D}, \mathrm{RA}$, and MS, is within an intron of $B A C H 2$, a gene involved in suppressing effector CD4 and CD8 T cell differentiation, while promoting regulatory $\mathrm{T}$ cell differentiation ${ }^{8,9}$ and $\mathrm{T}$ cell stemness ${ }^{10}$ (Fig. 3b). This variant falls preferentially within the accessible chromatin of naïve $\mathrm{T}$ cells and contacts the $B A C H 2$ promoter in naïve $\mathrm{T}$ cells ${ }^{23}$, with the risk allele reducing reporter expression (Fig. 2c). Collectively, these data suggest that disease-associated emVars that act in $\mathrm{T}$ cell regulatory regions regulate genes known to play a role in T cell signaling, differentiation, and function.

An emVar in accessible chromatin reduces $\boldsymbol{B} \boldsymbol{A C H} 2$ expression. We further characterized rs 729282038, as it displayed one of the strongest allelic biases in reporter activity in the MPRA (Fig. 2c). We first validated our MPRA results for rs72928038 using a luciferase assay in Jurkat 
T cells. We cloned a construct that contained either allele of rs 72928038 centered in $300 \mathrm{bp}$ of native genomic sequence upstream of the $B A C H 2$ promoter. We found that the risk allele (A) had lower luciferase activity (Supplementary Fig. 9a). There were two other statistically fine-mapped variants in the locus, rs10944479 (PICS probability 0.0458 in MS GWAS) and rs6908626 (posterior probability 0.0894 in MS GWAS) (in comparison, rs72928038 had posterior probability of 0.865 for MS GWAS). Neither of these variants were found to have allelic bias in the MPRA. These data suggest that, rs72928038 is the only variant in the credible set that alters regulatory activity (Fig. $4 \mathrm{a})$.

rs 729282038 is an eQTL specifically in naïve CD4, naïve CD8, and naïve regulatory T cells (but not other immune or T cell types) with the risk allele (A) associated with lower expression of $B A C H 2^{30}$. If rs72928038 acts through modulating enhancer activity in T cells to alter $B A C H 2$ expression, one may expect the variant to reside in accessible chromatin of $\mathrm{T}$ cells, but not of other cell types. Indeed, we found rs72928038 is located in accessible chromatin specifically in T cells, and not in B cells or monocytes (Fig. 4b). To assess differences in chromatin accessibility between alleles, we surveyed CD4 T cells from healthy donors who were heterozygous at rs 72928038 and observed the non-risk allele $(\mathrm{G})$ to be preferentially present in accessible chromatin (Fig. 4c; Supplementary Table 11). The 328 bp region surrounding rs 72928038 is annotated as a candidate cis-regulatory element by ENCODE (EH38E2485452) 31 and the risk allele is predicted to disrupt binding motifs for ETS or STAT family TFs (Fig. 4b; Supplementary Table 5). Furthermore, based on published promoter-capture HiC data, the region surrounding rs 72928038 physically interacts with the $B A C H 2$ promoter specifically in naïve $\mathrm{T}$ cells, but not other immune cell types (Supplementary Fig. 9b), suggesting that the risk allele of rs 72928038 regulates $B A C H 2$ expression in T cells through reducing cis-regulatory activity. 
We next sought more direct functional evidence for the role of rs 72928038 in altering $B A C H 2$ expression. To do this, we used a cytosine base editor along with a guide RNA targeting rs 72928038 to introduce the risk allele into the native genomic context in Jurkat T cells, which are homozygous for the non-risk allele (Methods) ${ }^{32}$ (Fig. 4d and Supplementary Fig. 10). Within the pool of nucleofected cells, we found $95 \%$ edited cells, with a range of bases edited in the editing window including specific edits of the variant of interest. To assess the effect of the risk variant on $B A C H 2$ expression, we used PrimeFlow, which uses in situ hybridization of antisense probes to the $\mathrm{BACH} 2$ transcript, followed by signal amplification, fluorescent labeling, and fluorescence-activated cell sorting to isolate cells that have either high or low $B A C H 2$ expression (Supplementary Fig. 10) $)^{6,7}$. For each bin, we sequenced the rs72928038 region and compared the prevalence of amplicons containing the edited risk allele to those without edits, finding that the risk variant reduces $B A C H 2$ expression (Fig. 4e, left). However, since unedited cells were only $5 \%$ of the cell population, the estimated expression levels of $B A C H 2$ in the WT cells were variable leading to unstable estimates of effect. To address this, we created a second condition with cells nucleofected with base editor and either a guide RNA targeting rs 72928038 or a safe harbor sequence, and then combined these cells at a 50/50 ratio, again finding that the baseedited risk variant confers reductions in $B A C H 2$ expression (Fig. 4e, right; Supplementary Fig. 10). Together, these experiments show that the rs 72928038 risk allele reduces the expression of $B A C H 2$ in a human $\mathrm{T}$ cell line.

\section{Deletion of the orthologous non-coding element containing rs72928038 in mice leads to} reduced expression of $\mathbf{T}$ cell stemness genes. To investigate the phenotypic effects of the regulatory region containing rs72928038 in primary naïve T cells, ideally one would isolate 
naïve $\mathrm{T}$ cells from secondary lymphoid organs, where they reside in $\mathrm{T}$ cell zones awaiting activation by an antigen presenting cell. Since these secondary lymphoid organs are easily harvested from mice, we explored the use of mice to study the effect of rs 72928038 on naïve T cells. We first assessed conservation between human and mouse at the site of the variant. Through synteny analysis of the locus between human and mouse, we found that the variant exists on mouse chromosome 4 within an intron of Bach2, similar to its position with respect to $B A C H 2$ in the human genome (Fig. 5a). The 328 bp human cCRE containing rs 72928038 is $51.2 \%$ conserved between species, with especially high conservation in the 16 bps surrounding rs 72928038 (Fig. 5a). Additionally, at the orthologous region, mouse T cells have accessible chromatin, H3K27ac deposition, and we found both ETS1 and STAT TFs bind (Supplementary Fig. 11a) ${ }^{33}$, consistent with the epigenetic profile observed in human T cells. Based on these findings, we created a mouse line containing an 18bp deletion of the non-coding region overlapping the variant using CRISPR-mediated genome editing (Bach2 ${ }^{18 \mathrm{del}}$; Fig. 5b, Supplementary Fig. 11a).

Using these mice, we performed experiments to determine if primary mouse naïve $\mathrm{T}$ cells containing the deletion had reduced Bach 2 expression and altered expression of other important genes that play a role in T cell biology. Bach2-ablated mice have previously been shown to have aberrant CD8 T cell activation ${ }^{8}$. To assess whether deletion of the variant alters naïve CD8 T cell phenotypes in mice, we sorted naïve CD8 T cells from Bach2 ${ }^{18 \mathrm{del}}$ and WT mice and analyzed their transcriptomes via BRB-seq ${ }^{34}$. We found Bach2 ${ }^{18 \mathrm{del}}$ naïve CD8 T cells to have altered transcriptional programs according to principal components analysis (PCA) and reduced Bach2 expression compared to WT littermates (Fig. 5c and d). Through differential expression analysis, we found 47 differentially expressed genes (Fig. 5e; Supplementary Table 12). Genes more 
highly expressed in Bach2 ${ }^{18 \mathrm{del}}$ naïve CD8 T cells were enriched for KLRG1 $1^{\text {lo }}$ effector CD8 T cell gene sets (Supplementary Table 13), as well as for a gene set in which CD8 T stem-like memory cells (Tscms) were perturbed with CRISPR/Cas9 targeted against Bach2 (Fig. 5f; Supplementary Fig. $11 \mathrm{~b})^{10}$. We found $66 \%$ of the differentially expressed genes from Bach $2^{18 \text { del }}$ naïve CD8 T cells have the same directionality in Bach2 guide RNA targeted Tscms (Supplementary Fig. 11c). Similar to Bach2-perturbed Tscms, Bach2 ${ }^{18 \mathrm{del}}$ naïve CD8 T cells had significantly reduced CD62L surface expression (Fig. 5g), concomitant with a reduction in Lefl and Myb expression (Fig. 5h); these TFs are required to maintain stemness of naïve T cells and Tscms, and are downregulated during effector $\mathrm{T}$ cell differentiation ${ }^{10,35-37}$. In addition, Bach2 ${ }^{18 \mathrm{del}}$ naïve CD8 T cells showed a reduction in Elf4 and a significant upregulation of ribosomal protein mRNAs, both indications of early T cell stimulation ${ }^{38,39}$ (Fig. 5e and h). Bach2 ${ }^{18 \mathrm{del}}$ naïve CD8 T cells also had reduced expression of Pten and Itch, both negative regulators of signaling that are required for suppressing effector $\mathrm{T}$ cell differentiation ${ }^{40,41}$ (Fig. 5h). Thus, deletion of the orthologous non-coding region containing rs 72928038 in mice leads to reduced features of naïve T cell stemness and indications of early $\mathrm{T}$ cell activation.

\section{DISCUSSION}

Identifying mechanisms that drive genetic risk for autoimmunity and other complex phenotypes remains a substantial challenge. Most autoimmune genetic associations have many variants in tight LD to the lead variant ${ }^{3}$, and the majority of these variants have not been functionally characterized using a systematic approach within disease-relevant cell types. Here, using a combination of MPRA and T cell chromatin accessibility, we identify 60 variants associated with five autoimmune diseases that enrich 57.8-fold for causal variants according to statistical fine- 
mapping. Collectively, these data demonstrate that this combination of methods serves as a robust prioritization scheme for identifying causal variants for disease associations. Many GWAS loci with T-GWAS emVars are near genes that regulate T cell function, or those that are known to dysregulate $\mathrm{T}$ cells in the context of autoimmune disease. One of the variants with high allelic bias in MPRA was rs72928038, a variant in an intron of $B A C H 2$. We found the rs 72928038 risk allele was less present in chromatin accessibility data from T cells harvested from humans heterozygous for rs 72928038 , and base editing of the rs72928038 risk allele in a human $\mathrm{T}$ cell line reduced $B A C H 2$ expression. We also engineered mice that have an $18 \mathrm{bp}$ deletion overlapping orthologous rs72928038 (Bach2 ${ }^{18 \mathrm{del}}$ ), and found that their naïve CD8 T cells had reduced Bach2 expression, as well as reduced expression of transcriptional regulators of $\mathrm{T}$ cell stemness and indications of early $\mathrm{T}$ cell activation.

By applying MPRA, we substantially enriched for statistically fine-mapped GWAS variants, an enrichment that was further magnified when combining these data with chromatin accessibility data from $T$ cells. Combining MPRA and accessible chromatin data was an effective strategy, possibly because chromatin accessibility, which provides an endogenous measure of cis-regulatory activity from relevant cell types, acts as a stringency filter for MPRA, which is plasmid-based. This strategy was further supported by an increase in sensitivity for identifying credible set variants from $18.4-19.7 \%$ to $23.1-25.5 \%$ while maintaining a specificity of $81.1-91.7 \%$. Other prioritization methodologies could also be applied in tandem such as allelespecific ATAC-seq ${ }^{12}$, CRISPR-inhibition ${ }^{7,42}$, and SELEX ${ }^{43}$, among others. However, requiring a variant to score for multiple methodologies may substantially increase type II error, as different methods tend to test different genomic features and have variable signal-to-noise ratios ${ }^{6}$. Thus, combining data from orthogonal tests of variant action with high signal-to-noise ratios, such as 
MPRA and accessible chromatin, could provide a reasonable balance between sensitivity and specificity $^{6}$. Because perturbational and statistical fine-mapping are still imperfect approaches, discovery of causal variants still requires further mechanistic evaluation, ideally within systems that recapitulate the (patho)-physiological environment of the disease.

While we discovered emVars for $\sim 31 \%$ of GWAS loci studied, there are a variety of reasons why many loci did not contain an identified emVar. We found emVars to be enriched in TSS regions, thus this methodology may have increased sensitivity for variants that alter promoter activity. We performed the MPRA in unstimulated conditions, although variants may disrupt TFs that are downstream of signaling cascades following $\mathrm{T}$ cell stimulation or differentiation into specific effector cell subsets (e.g., Th1, Th2, Th17, Treg, Tfh). Stimulation with various ligands in eQTL studies has been crucial for identifying variants that were otherwise inactive at baseline ${ }^{44-46}$. Other cell types also likely play a role in these autoimmune diseases; beta cell accessible chromatin is enriched for T1D-associated variants, especially after stimulation with pro-inflammatory cytokines ${ }^{47}$, B cell accessible chromatin enriches for MSassociated variants $^{48}$, skin cell accessible chromatin enriches for psoriasis ${ }^{49}$, and intestinal CAGE data enriches for IBD-associated variants ${ }^{50}$. Variants in GWAS loci may have roles beyond disrupting cis-regulatory elements, such as coding mutations, altering the activity of untranslated regions (UTRs), or promoting alternative splicing. These actions are unlikely to be identified by MPRAs designed to test how variants modulate regulatory region activity, but alternative massively parallel methodologies have been created to address how variants may alter UTR function and alternative splicing ${ }^{51,52}$. Thus, applying the prioritization scheme of MPRA + accessible chromatin and other methodologies to a wider range of cell types and stimulation conditions could unveil additional likely causal variants. 
Using our MPRA and accessible chromatin prioritization scheme, we found variants in GWAS loci that were highly relevant to T cell biology, including rs 72928038 in the $B A C H 2$ locus. We selected rs 72928038 for further mechanistic studies due to its high allelic bias in MPRA and the strength of genetic and epigenetic evidence supporting the variant. Through base editing the variant in Jurkat $\mathrm{T}$ cells and deleting the orthologous region surrounding the variant in mice, we found rs72928038 and the regulatory region in which it is contained altered T cell $B A C H 2$ expression. Similar to Bach2-deficient CD8 stem-like memory cells from a separate study ${ }^{10}$, we observed that Bach2 $2^{18 \text { del }}$ naïve CD 8 T cells have reductions in stem-associated TFs Lef1, Myb, antiproliferative TF Elf4, signaling attenuators Pten and Itch, and a reduction in surface expression of CD62L. We also found an increase in expression of ribosomal genes, indicative of early $\mathrm{T}$ cell stimulation ${ }^{39}$. Consistent with increased activation, we saw enrichments of effector CD8 $\mathrm{T}$ cell gene programs. Collectively these data suggest that the Bach2 ${ }^{18 \mathrm{del}}$ naïve CD8 T cells may have a reduced threshold for activation. However, Bach2 ${ }^{18 \mathrm{del}}$ naïve CD8 T cells do not appear to have phenotypes of fully differentiated effector cells (e.g., increased expression of $G z m b, K l r g l$ and $C d 44$ ), possibly due to only partial Bach2 reduction mediated by removing only a single regulatory element as opposed to deletion of the gene. Indeed several TFs have been noted to act in a graded manner to promote transcription and cell fate during the differentiation of CD8 T cells ${ }^{53,54}$, and mice heterozygous for the deletion of Bach2 show intermediate effects on $\mathrm{T}$ cell differentiation between WT and homozygous mice ${ }^{9}$. Other than naïve T cells, further dissection will be required to understand the effects of rs 72928038 on subsets of $\mathrm{T}$ cells that are known to require BACH2 for fate determination, such as Tscms, memory T cells, and Tregs ${ }^{8-10}$. Thus, organismic models, such as the Bach $2^{18 \mathrm{del}}$ mice, provide 
rare insight into the physiological effects of variants and their regulatory elements within living systems.

In summary, this work provides a scalable and high-yield prioritization scheme to identify likely causal variants at high specificity. We find 60 likely causal variants that have significant evidence for acting in T cells, and direct evidence of a variant that reduces Bach2 expression and transcriptional hallmarks of naïve $\mathrm{T}$ cells and $\mathrm{T}$ cell stemness. Together, this work demonstrates a clear path for addressing the long-term obstacle of defining causal variants for complex traits and their effects on gene regulation and cellular and organismal functions.

\section{METHODS}

\section{Cell lines}

For MPRA, luciferase, and base editing experiments, we used low passage aliquots of the Jurkat T cell line (ATCC TIB-152 ${ }^{\mathrm{TM}}$ ), maintaining the culture under 20 passages. Cells were grown at $37^{\circ} \mathrm{C}$ maintaining cultures between $1 \times 10^{5}$ and $1 \times 10^{6}$ cells per $\mathrm{mL}$.

\section{Study subjects}

The study was performed in accordance with protocols approved by the institutional review board at Partners (Brigham and Women's Hospital, Massachusetts General Hospital, DanaFarber Cancer Institute, Boston, USA) and Broad Institute (USA) Research Ethics Committee, as well as the Feinstein Institute for Medical Research, Northwell Health institutional review board (Manhasset New York, USA). All donors provided written informed consent for the genetic research studies and molecular testing. Healthy donors were recruited from the Sisters of Lupus Erythematosus patients (SisSLE) Research Study based in Manhasset, NY, and the Boston-based PhenoGenetic project, a resource of healthy subjects. 


\section{GWAS data}

Lead SNPs were obtained from GWAS for T1D ${ }^{20}, \mathrm{RA}^{22}$, psoriasis $^{21}, \mathrm{IBD}^{17}$, and MS ${ }^{18,19}$. We collected 578 GWAS lead SNPs from these studies, representing 531 distinct GWAS loci. We identified all proxy SNPs $\left(\mathrm{r}^{2} \geq 0.8\right)$ for each lead SNP based on 1000 Genomes Phase 3 European subset. Proxy SNPs were identified using PLINK v1.90b3.32 ${ }^{60}$ (www.coggenomics.org/plink/2.0/) with parameters --r2 --ld-window-kb 2000 --ld-window 999999 --ldwindow-r2 0.8 . There were 20792 total proxy SNPs across the 578 GWAS loci (18324 unique proxy SNPs across these 531 distinct GWAS loci).

\section{MPRA}

MPRA oligo synthesis and cloning was adapted from refs ${ }^{6,13}$. Each allele was tagged with an average of $\sim 1,000$ DNA barcodes. Oligos were synthesized by Agilent Technologies containing $170 \mathrm{bp}$ of genomic context and $15 \mathrm{bp}$ of adapter sequence at either end (5'-

ACTGGCCGCTTGACG[170 bp oligo]CACTGCGGCTCCTGC-3'; Supplementary Table 14; $200 \mathrm{bp}$ total). $20 \mathrm{bp}$ barcodes and additional adapter sequences were added by performing 28 emulsion PCR reactions, each $50 \mu \mathrm{L}$ in volume containing $1.86 \mathrm{ng}$ of oligo, $25 \mu \mathrm{L}$ of Q5

NEBNext MasterMix (NEB, M0541S), 1 unit Q5 HotStart polymerase (NEB, M0493S), $0.5 \mu \mathrm{M}$ MPRA_v3_F and MPRA_v3_20I_R primers (Supplementary Table 14) and 2 ng BSA (NEB, B9000). PCR master mix was emulsified by vortexing with $220 \mu \mathrm{L}$ Tegosoft DEC (Evonik), $60 \mu \mathrm{L}$ ABIL WE (Evonik) and $20 \mu \mathrm{L}$ mineral oil (Sigma, M5904) per $50 \mu \mathrm{L}$ PCR reaction at $4{ }^{\circ} \mathrm{C}$ for $5 \mathrm{~min} .50 \mu \mathrm{L}$ of emulsion mixture was added to each well of a 96 -well plate and cycled with the following conditions; $95^{\circ} \mathrm{C}$ for $30 \mathrm{~s}, 15$ cycles of $\left(95^{\circ} \mathrm{C}\right.$ for $20 \mathrm{~s}, 60^{\circ} \mathrm{C}$ for $10 \mathrm{~s}, 72^{\circ} \mathrm{C}$ for $15 \mathrm{~s}$ ), $72^{\circ} \mathrm{C}$ for $5 \mathrm{~min}$. Amplified emulsion mixture was broken and purified by adding $1 \mathrm{~mL}$ of 2-butanol (VWR, AA43315-AK), $50 \mu \mathrm{L}$ of AMPure XP SPRI (Beckman Coulter, A63881) 
and $80 \mu \mathrm{L}$ of binding buffer $(2.5 \mathrm{M} \mathrm{NaCl}, 20 \%$ PEG-8000) per $350 \mu \mathrm{L}$ of emulsion mix and vigorously vortexed, followed by incubation for $10 \mathrm{~min}$ at room temperature. Broken emulsion/butanol mixture was spun at $2900 \times g$ for $5 \mathrm{~min}$ and the butanol phase was discarded. The aqueous phase was placed on a magnetic rack for 20 min prior to aspiration. Remaining beads were washed once with 2-butanol, three times with $80 \%$ EtOH and eluted in EB (Qiagen, 19086) to yield the barcoded oligo pool.

To create our mpra $\Delta$ orf library, barcoded oligos were inserted into SfiI digested pGL4.23 $\Delta x$ ba $\Delta$ luc by Gibson Assembly (NEB, E2611) using $1.1 \mu \mathrm{g}$ of oligos and $1 \mu \mathrm{g}$ of digested vector in a $40 \mu \mathrm{L}$ reaction incubated for $60 \mathrm{~min}$ at $50^{\circ} \mathrm{C}$ followed by AMPure XP SPRI purification and elution in $20 \mu \mathrm{L}$ of EB. Half of the ligated vector was then transformed into $200 \mu \mathrm{L}$ of Endura ${ }^{\mathrm{TM}}$ ElectroCompetent E. coli (Lucigen, 60242-2) by electroporation (1.8 kV, $600 \Omega, 10 \mu \mathrm{F})$. Electroporated bacteria were immediately split into eight $1 \mathrm{~mL}$ aliquots of SOC (NEB, B9020S) and recovered for $1 \mathrm{~h}$ at $37^{\circ} \mathrm{C}$ then independently expanded in $20 \mathrm{~mL}$ of LB supplemented with $100 \mu \mathrm{g} / \mathrm{mL}$ of carbenicillin (EMD, 69101-3) on a floor shaker at $37^{\circ} \mathrm{C}$ for $6.5 \mathrm{~h}$. After outgrowth, aliquots were pooled prior to plasmid purification (Qiagen, 12963). For each of the aliquots, we plated serial dilutions after SOC recovery and estimated a library size of $\sim 4 \times 10^{7}$ CFUs, representing $\sim 1000$ barcodes per allele.

To insert minP and GFP ORF, $20 \mu \mathrm{g}$ of mpra: $\Delta$ orf plasmid was linearized with AsiSI (NEB, R0630S) and 1x CutSmart buffer (NEB, B7204S) in a $500 \mu \mathrm{L}$ volume for $3.5 \mathrm{~h}$ at $37^{\circ} \mathrm{C}$, followed by bead inactivation for $20 \mathrm{~m}$ at $80{ }^{\circ} \mathrm{C}$ and SPRI cleaning. An amplicon containing minP, the GFP open-reading frame, and a partial 3' UTR was then inserted by Gibson assembly using $10 \mu \mathrm{g}$ of AsiSI linearized mpra $\Delta$ orf plasmid, $33 \mu \mathrm{g}$ of the minP/GFP amplicon in $400 \mu \mathrm{L}$ of total volume for $90 \mathrm{~min}$ at $50{ }^{\circ} \mathrm{C}$ followed by a $1.5 \times$ beads/sample SPRI purification. The total 
recovered volume was digested a second time to remove remaining uncut vectors by incubation with AsiSI in a $100 \mu \mathrm{L}$ reaction for $6 \mathrm{~h}$ at $37^{\circ} \mathrm{C}$ followed by Ampure XP purification and elution with $55 \mu \mathrm{L}$ of Buffer EB.

$10 \mu \mathrm{L}$ of the mpra:minP:gfp plasmid was electroporated $(1.8 \mathrm{kV}, 600 \Omega, 10 \mu \mathrm{F})$ into $200 \mu \mathrm{L}$ of Endura cells. Electroporated bacteria was split across six tubes and each recovered in $2 \mathrm{~mL}$ of SOC for $1 \mathrm{~h}$ at $37^{\circ} \mathrm{C}$ then added to $500 \mathrm{~mL}$ of LB with $100 \mu \mathrm{g} / \mathrm{mL}$ of carbenicillin and grown for $9 \mathrm{~h}$ at $37^{\circ} \mathrm{C}$ prior to plasmid purification (Qiagen, 12991). The plasmid prep was then normalized to $1 \mu \mathrm{g} / \mu \mathrm{L}$ to generate the final mpra:minP:gfp library used for transfection delivery.

For all transfections, cells were grown to a density of $\sim 1 \times 10^{6}$ cells $/ \mathrm{mL}$, and $1 \times 10^{8}$ cells were used for each experiment. Cells were collected by centrifugation at $300 \times g$ and eluted in $1 \mathrm{~mL}$ of RPMI with $100 \mu \mathrm{g}$ of mpra:minP:gfp library. Electroporation was performed in $100 \mu \mathrm{L}$ volumes with the Neon transfection system (Life Technologies) applying three pulses of 1600V for $10 \mathrm{~ms}$ each into Jurkat T cells. Using separate control transfections, we achieved transfection efficiencies of $40-60 \%$ for all replicates. Cells were allowed to recover in $200 \mathrm{~mL}$ in RPMI with $15 \%$ FBS for $24 \mathrm{~h}$ before being collected by centrifugation, washed once with PBS, collected and frozen at $-80^{\circ} \mathrm{C}$.

Total RNA was extracted from cells using QIAGEN Maxi RNeasy (QIAGEN, 75162) following the manufacturer's protocol including the on-column DNase digestion. A second DNase treatment was performed on the purified RNA using $5 \mu \mathrm{L}$ of Turbo DNase (Life Technologies, AM2238) with buffer, in $750 \mu \mathrm{L}$ of total volume for $1 \mathrm{~h}$ at $37^{\circ} \mathrm{C}$. The digestion was stopped with the addition of $7.5 \mu \mathrm{L} 10 \% \mathrm{SDS}$ and $75 \mu \mathrm{L}$ of $0.5 \mathrm{M}$ EDTA followed by a 5min incubation at $70{ }^{\circ} \mathrm{C}$. The total reaction was then used for pulldown of GFP mRNA. Water was added to the DNase-digested RNA to bring the total volume to $898 \mu \mathrm{L}$ to which $900 \mu \mathrm{L}$ of 
20X SSC (Life Technologies, 15557-044), $1800 \mu \mathrm{L}$ of Formamide (Life Technologies, AM9342) and $2 \mu \mathrm{L}$ of $100 \mu \mathrm{M}$ biotin-labeled GFP probe (GFP_BiotinCapture_1-3, IDT, Supplementary Table 14) were added and incubated for $2.5 \mathrm{~h}$ at $65^{\circ} \mathrm{C}$. Biotin probes were captured using $400 \mu \mathrm{L}$ of pre-washed Streptavidin beads (Life Technologies, 65001) eluted in $500 \mu \mathrm{L}$ of 20X SSC. The hybridized RNA/probe bead mixture was agitated on a nutator at room temperature for $15 \mathrm{~min}$. Beads were captured by magnet and washed once with $1 \times$ SSC and twice with $0.1 \times$ SSC. Elution of RNA was performed by the addition of $25 \mu \mathrm{L}$ water and heating of the water/bead mixture for $2 \mathrm{~min}$ at $70^{\circ} \mathrm{C}$ followed by immediate collection of eluent on a magnet. A second elution was performed by incubating the beads with an additional $25 \mu \mathrm{L}$ of water at $80{ }^{\circ} \mathrm{C}$. A final DNase treatment was performed in $50 \mu \mathrm{L}$ total volume using $1 \mu \mathrm{L}$ of Turbo DNase with addition of the buffer incubated for $60 \mathrm{~min}$ at $37^{\circ} \mathrm{C}$ followed by inactivation with $1 \mu \mathrm{L}$ of $10 \% \mathrm{SDS}$ and purification using RNA clean SPRI beads (Beckman Coulter, A63987).

First-strand cDNA was synthesized from half of the DNase-treated GFP mRNA with SuperScript III and a primer specific to the 3' UTR (MPRA_v3_Amp2Sc_R, Supplementary Table 14) using the manufacturer's recommended protocol, modifying the total reaction volume to $40 \mu \mathrm{L}$ and performing the elongation step at $47^{\circ} \mathrm{C}$ for $80 \mathrm{~min}$. Single-stranded cDNA was purified by SPRI and eluted in $30 \mu \mathrm{L}$ EB.

To minimize amplification bias during the creation of cDNA tag sequencing libraries, samples were amplified by qPCR to estimate relative concentrations of GFP cDNA using $1 \mu \mathrm{L}$ of sample in a $10 \mu \mathrm{L}$ PCR reaction containing $5 \mu \mathrm{L}$ Q5 NEBNext master mix, $1.7 \mu \mathrm{L}$ SYBR Green I diluted 1:10,000 (Life Technologies, S-7567) and 0.5 $\mu \mathrm{M}$ of TruSeq_Universal_Adapter and MPRA_Illumina_GFP_F primers (Supplementary Table 14). Samples were amplified with the following qPCR conditions: $95^{\circ} \mathrm{C}$ for $20 \mathrm{~s}, 40$ cycles $\left(95^{\circ} \mathrm{C}\right.$ for $20 \mathrm{~s}, 65^{\circ} \mathrm{C}$ for $20 \mathrm{~s}, 72^{\circ} \mathrm{C}$ for 
$30 \mathrm{~s}$ ), $72^{\circ} \mathrm{C}$ for $2 \mathrm{~min}$. The number of cycles for sample amplification was $1-n$ (the number of cycles it took for each sample to pass the threshold) from the qPCR. To add Illumina sequencing adapters, $10 \mu \mathrm{L}$ of cDNA samples and mpra:minP:gfp plasmid control (diluted to the qPCR cycle range of the samples) were amplified using the reaction conditions from the qPCR scaled to $50 \mu \mathrm{L}$, excluding SYBR Green I. Amplified cDNA was SPRI purified and eluted in $40 \mu \mathrm{L}$ of EB. Individual sequencing barcodes were added to each sample by amplifying the entire $40 \mu \mathrm{L}$ elution in a $100 \mu \mathrm{L}$ Q5 NEBNext reaction with $0.5 \mu \mathrm{M}$ of TruSeq_Universal_Adapter primer and a reverse primer containing a unique 8 bp index (Illumina_Multiplex, Supplementary Table 14) for sample demultiplexing post-sequencing. Samples were amplified at $95^{\circ} \mathrm{C}$ for $20 \mathrm{~s}$, six cycles $\left(95^{\circ} \mathrm{C}\right.$ for $20 \mathrm{~s}, 64^{\circ} \mathrm{C}$ for $30 \mathrm{~s}, 72^{\circ} \mathrm{C}$ for $\left.30 \mathrm{~s}\right), 72^{\circ} \mathrm{C}$ for $2 \mathrm{~min}$. Indexed libraries were SPRI purified and pooled according to molar estimates from Agilent TapeStation quantifications. Samples were sequenced using $1 \times 30$ bp chemistry on a NextSeq 2000 (Illumina).

To determine oligo/barcode combinations within the MPRA pool, Illumina libraries were prepared from the mpra $\Delta$ orf plasmid library by performing five separate amplifications with $200 \mathrm{ng}$ of plasmid in a $100 \mu \mathrm{L}$ Q5 NEBNext PCR reaction containing $0.5 \mu \mathrm{M}$ of TruSeq_Universal_Adapter and MPRA_v3_TruSeq_Amp2Sa_F primers (Supplementary Table 14) with the following conditions: $95^{\circ} \mathrm{C}$ for $20 \mathrm{~s}, 6$ cycles $\left(95^{\circ} \mathrm{C}\right.$ for $20 \mathrm{~s}, 62^{\circ} \mathrm{C}$ for $15 \mathrm{~s}, 72^{\circ} \mathrm{C}$ for $30 \mathrm{~s}$ ), $72^{\circ} \mathrm{C}$ for $2 \mathrm{~min}$. Amplified material was SPRI purified using a $0.6 \times \mathrm{bead} / \mathrm{sample}$ ratio and eluted with $30 \mu \mathrm{L}$ of EB. Sequencing indexes were then attached using $20 \mu \mathrm{L}$ of the eluted product and the same reaction conditions as for the tag-seq protocol, except the number of enrichment cycles was lowered to 5 . Samples were molar pooled and sequenced using $2 \times 150 \mathrm{bp}$ chemistry on an Illumina NextSeq 2000.

\section{MPRA analysis}


Barcode sequencing results from the MPRA were analyzed as previously described ${ }^{13}$. Briefly, the sum of the barcode counts for each oligo was provided as input to DESeq2 and replicates were median normalized followed by an additional normalization of the RNA samples to center the RNA/DNA activity distribution over a $\log _{2}$ fold change of zero ${ }^{72}$. Oligos showing differential expression relative to the plasmid input were identified by modeling a negative binomial distribution with DESeq2 and applying a false discovery rate (FDR) threshold of $1 \%$. For sequences that displayed significant MPRA activity, a paired two-sided Student's $t$-test was applied on the log-transformed RNA/plasmid ratios for each experimental replicate to test whether the reference and alternate allele had similar activity. An FDR threshold of 10\% was used to identify SNPs with a significant difference in MPRA activity between alleles (emVars).

\section{Genomic data integration and enrichment analyses}

DHS data across 733 samples was obtained from ${ }^{4}$. Pre-processed DHS peaks lifted to hg19 were downloaded from https://zenodo.org/record/3838751\#.X_IA7-1Kg6U. For each of 261 unique cell types, DHS peaks were merged across replicates of the same cell type. To assess enrichment between DHS sites for each cell type and MPRA data, we counted the number of MPRA variants with regulatory activity (i.e., pCRE) overlapping the genomic interval bed file for each cell type. We then constructed a $2 \times 2$ contingency table based on whether the MPRA SNP showed regulatory activity (i.e., pCRE) or had no regulatory activity, and whether the SNP intersected a genomic interval or not. P values were calculated based on a two-sided Fisher's exact test.

Multiple testing correction was performed using Bonferroni correction by taking an alpha of 0.05 divided by the number of unique cell types tested $(n=261)$.

Histone ChIP-seq data were obtained from the ENCODE project for all available human CD4 positive alpha-beta $\mathrm{T}$ cell samples. For each cell type, all available ChIP-seq peak call sets 
for H3K4me1, H3K4me3, H3K27ac, and H3K36me3 aligned to hg19 were downloaded in bed file format. If multiple replicates were available, peaks call sets were merged using the merge function in BEDTools v2.26.061. Human CAGE-based enhancer sequences were downloaded from https://fantom.gsc.riken.jp/5/datafiles/latest/extra/Enhancers/ in bed file format.

chromHMM annotations in the 18 chromatin state model were obtained for primary $\mathrm{T}$ cells from peripheral blood (E034) from the Roadmap Epigenomics Project

(https://egg2.wustl.edu/roadmap/data/byFileType/chromhmmSegmentations/ChmmModels/core K27ac/jointModel/final/) in bed file format. To assess enrichment between each of these genomic datasets and MPRA data, we counted the number of emVar SNPs overlapping the genomic interval bed file. We then constructed a 2 × 2 contingency table based on whether the SNP showed MPRA activity (i.e., emVar) or had no MPRA activity, and whether the SNP intersected a genomic interval or not. P values were calculated based on a two-sided Fisher's exact test. Multiple testing correction was performed using Bonferroni correction by taking an alpha of 0.05 divided by the number of genomic annotations tested.

\section{Transcription factor enrichment}

To calculate the predicted effect of each MPRA variant on TF binding, we applied motifbreakR version 2.4. $0^{62}$. For each single nucleotide substitution in the MPRA data, we calculated predicted TF binding scores for the reference and alternate alleles. We used the sum of log probabilities approach in motifbreakR, applied to all TF position-weighted matrices in HOCOMOCO v10 63 . All MPRA SNPs with a difference in TF binding scores between the reference and alternate alleles at $P<1 \times 10^{-5}$ were considered to be significant.

To assess overlaps between MPRA SNPs and TF binding motifs derived from ChIP-seq, we applied HOMER v4.1064. For each emVar, we generated sequences in a \pm 100 bp window 
around the emVar SNP. We then generated background sequences in a $\pm 100 \mathrm{bp}$ window around all variants tested in the MPRA. To calculate enrichments, we applied findMotifGenome.pl in HOMER with -size 200; all other default parameters were used. Enrichments were tested against the library of all known TF motifs available in HOMER.

\section{ATAC-seq skew and QTL enrichment}

To assess enrichment of MPRA SNPs at sites of allelic skew in open chromatin regions, we downloaded significant allelic skew SNPs from Calderon et al. ${ }^{12}$. We used all available unstimulated hematopoietic cell types. For SNPs with evidence of skew across multiple samples or cell types, we summed the reference and alternate allele counts for that SNP across the cell types. To assess enrichment of MPRA SNPs at ATAC-QTLs, we downloaded primary T-cell ATAC-QTLs from ${ }^{65}$.

\section{In silico predictions of effect of regulatory activity}

We applied deltaSVM v1.3 $3^{66}$ to predict the effect of MPRA SNPs on regulatory activity. This method uses a classifier (gkm-SVM) to encode cell-specific regulatory sequence vocabularies, and then subsequently deltaSVM quantifies the effect of a SNP as the change in gkm-SVM score). To apply deltaSVM, we downloaded pre-computed gkm-SVM weights derived from ENCODE2 enhancers in naïve CD4 T cells. We then used deltasvm.pl from the software developers to calculate deltaSVM scores for each emVar. Default parameters were used throughout.

\section{PICS fine-mapping and enrichment analyses}

For each GWAS locus, PICS ${ }^{1}$ was applied to all SNPs in LD $\left(r^{2} \geq 0.8\right)$ to the lead SNP at based on the 1000 Genomes Phase 3 European subset using PLINK v1.90b3.32 with parameters --r2 -ld-window-kb 2000 --ld-window 999999 --ld-window-r2 0.8. GWAS association P values for 
lead SNPs were obtained from the EMBL GWAS catalog ${ }^{56}$ on August 10, 2020 ${ }^{56}$. If the same lead SNP was seen multiple times in the GWAS catalog for either the same disease or multiple diseases, the most significant lead SNP P value was used. Given long-range patterns of high LD, we excluded the human MHC locus (chr6:29691116-33054976 in hg19) and excluded any lead SNP where the most significant GWAS association P value did not reach $5 \times 10^{-8}$. In total, 512 GWAS loci were analysed. PICS fine-mapping posterior probabilities were calculated using a custom PERL script. Of note, in the scenario where a lead SNP was seen multiple times (either across the same disease or shared by different diseases), all proxy SNPs to the lead SNP were assigned based on the most significant lead SNP association P value, and PICS probabilities were calculated for both this lead SNP and its proxies.

We defined SNPs as being statistically fine-mapped based on either if it had a posterior probability greater than a given threshold, or if it was in a fine-mapping credible set. We used posterior probability thresholds of $\geq 0.01,0.05,0.1,0.2,0.3$, or 0.5 . We also calculated credible sets of fine-mapping variants. An X\% credible set is expected to contain the true causal variant $\mathrm{X} \%$ of the time. To generate credible sets, we greedily summed up the highest fine-mapping posterior probabilities at each locus until reaching a cumulative $\mathrm{X} \%$. We further required all credible set variants to have a fine-mapping posterior probability $\geq 0.01$.

To calculate enrichment of MPRA emVars in PICS fine-mapped SNPs, we determined for each MPRA variant whether it was a PICS statistically fine-mapped SNP (i.e., had a PICS probability greater than a given threshold) and whether the fine-mapped SNP showed MPRA activity (i.e., emVar). From this, we constructed $2 \times 2$ contingency tables. We then calculated variant-level enrichment $\left(E_{v}\right)$, represented as a risk ratio:

$$
E_{v}=\frac{\left(n_{v}^{M+P+}\right) /\left(n_{v}^{M+P+}+n_{v}^{M+P-}\right)}{\left(n_{v}^{M-P+}\right) /\left(n_{v}^{M-P+}+n_{v}^{M-P-}\right)}
$$


Here, $n_{v}$ refers to the number of variants. Superscript $M$ refers to whether the variant is an emVar $(M+$ if emVar, $M-$ otherwise $)$. Superscript $P$ refers to whether the SNP is a statistically fine-mapped SNP by PICS ( $P+$ if PICS fine-mapped, $P-$ otherwise). We can then construct a 2 x 2 contingency table with values $n_{v}^{M+P+}, n_{v}^{M+P-}, n_{v}^{M-P+}$, and $n_{v}^{M-P-}$. Statistical significance was calculated using a two-sided Fisher's exact test based off this 2 x 2 contingency table.

We similarly calculated enrichment of MPRA variants overlapping T cell DHS sites in PICS statistically fine-mapped SNPs. We determined for each MPRA variant whether it directly overlapped a T cell DHS site and whether it was a PICS fine-mapped SNP (i.e., had a PICS probability greater than a given threshold). We then calculated variant-level enrichment $\left(E_{v}\right)$ represented as a risk ratio:

$$
E_{v}=\frac{\left(n_{v}^{M+D+P+}\right) /\left(n_{v}^{M+D+P+}+n_{v}^{M+D+P-}\right)}{\left(n_{v}^{M-D-P+}+n_{v}^{M+D-P+}+n_{v}^{M-D+P+}\right) /\left(n_{v}^{M-D-P+}+n_{v}^{M+D-P+}+n_{v}^{M-D+P+}+n_{v}^{M-D-P-}+n_{v}^{M+D-P-}+n_{v}^{M-D+P-}\right)}
$$

Here, superscript $D$ refers to whether the variant is within a DHS site $(D+$ if $\operatorname{emVar}, D-$ otherwise) and superscript $M$ refers to whether the variant is an emVar $(M+$ if emVar, $M-$ otherwise). We can then construct a $2 \times 2$ contingency table with values $n_{v}^{M+D+P+}, n_{v}^{M+D+P-}$, $n_{v}^{M-D-P+}+n_{v}^{M+D-P+}+n_{v}^{M-D+P+}$, and $n_{v}^{M-D-P-}+n_{v}^{M+D-P-}+n_{v}^{M-D+P-} . \mathrm{P}$ values were calculated using a two-sided Fisher's exact test.

We also calculated sensitivity and specificity of the MPRA using PICS fine-mapping as the benchmark "ground truth." To do this, we tabulated for each locus, if there was a finemapped SNP (i.e., in X\% credible set) and whether the fine-mapped SNP showed MPRA activity (i.e., emVar). We can then calculate sensitivity at the locus level $\left(S E_{L}\right)$ :

$$
S E_{L}=\frac{\left(n_{L}^{M+P+}\right)}{\left(n_{L}^{M+P+}+n_{L}^{M-P+}\right)}
$$

And specificity $\left(S P_{L}\right)$ : 


$$
S P_{L}=\frac{\left(n_{L}^{M-P-}\right)}{\left(n_{L}^{M-P-}+n_{L}^{M+P-}\right)}
$$

Here, $n_{L}$ refers to the number of GWAS loci. Superscript $M$ refers to whether the variant is an emVar $(M+$ if emVar, $M-$ otherwise $)$. Superscript Prefers to whether the SNP is a statistically fine-mapped SNP by PICS ( $P+$ if PICS fine-mapped, $P-$ otherwise).

\section{T1D GWAS fine-mapping enrichment analysis}

For T1D GWAS, we obtained statistical fine-mapping credible sets from Onengut-Gumuscu et al. ${ }^{20}$. The main fine-mapping results from this study were used (Supplementary Table 1 of Onengut-Gumuscu et al.). We considered all T1D GWAS loci where an emVar was identified $(n=11)$. For each MPRA variant in these loci, we then determined whether it was in the finemapping study credible set and had a fine-mapping posterior probability above a specified threshold. If a SNP was not part of the fine-mapping because it was present in 1000 Genomes Project but not ImmunoChip (SNPs designated by "proxy" from the T1D study authors), the SNP was assigned the fine-mapping posterior probability of its closest ImmunoChip proxy. To assess enrichments for MPRA in the T1D statistical fine-mapping data, we constructed a 2 × 2 contingency table based on whether the SNP did or did not show MPRA activity (i.e., emVar), and whether the SNP was a statistically fine-mapped variant. Enrichments and P values were calculated as described above.

\section{Visualization of GWAS loci}

For visualization of gene tracks, bigWig files (Fig. 3a, 3b, Supplementary Fig. 8a and 8b) were downloaded from ENCODE. For cell types with multiple bigWig tracks, these were merged using bigWigMerge in the UCSC genome browser software suite ${ }^{76}$. bigWig tracks were then loaded into the UCSC genome browser (hg19). Track heights were adjusted to the maximum height of all tracks in a given viewing window. Gene transcripts are based on default UCSC 
genome browser gene annotations. To calculate the DHS score, the DHS sequencing depth in a \pm 10 bp window around each MPRA SNP was calculated using the multiBigwigSummary command in deepTools v3.5.0 $0^{77}$ with default options. The DHS score plot shows the maximum DHS signal observed across each of the T cell types. PCHiC loops for Fig.s. 3a, 3b and Supplementary Fig. 9 were downloaded from https://www.chicp.org/. The visualized loops were selected from those with a CHiCAGO score $\geq 5$ are shown.

\section{Luciferase Assay}

Firefly luciferase reporter constructs (pGL4.24) were generated by cloning the 300 nucleotide genomic region centered on rs72928038 (rs72928038_luc_G and rs72928038_luc_A, Supplementary Table 14) of interest upstream of the $B A C H 2$ promoter (Bach2 promoter_luc Supplementary Table 14) by using BgIII and XhoI sites. The firefly luciferase constructs (500 ng) were nucleofected with a pRL-SV40 Renilla luciferase construct (50 ng) into 2 x $10^{6}$ Jurkat cells by using the Neon nucleofection system (Invitrogen) using the program 1600V, 3 pulses, 10ms. After 48 h, luciferase activity was measured by Dual-Glo Luciferase assay system (Promega) according to the manufacturer's protocol. For each sample, the ratio of firefly to Renilla luminescence was measured and normalized to the empty pGL4.24 construct. Two separate biological replicates with at least 3 technical replicates per rs72928038 allele were conducted. For comparison of luminescence conferred by rs72928038 risk and non-risk alleles in the luciferase assay, we used a two-sided Student's $t$-test.

\section{Base-editing and PrimeFlow}

Base editor mRNA (evoCDAmax-SpCas9-NG) was provided by TriLink Biotechnologies, and was transcribed in vitro from PCR product using full substitution of 5-methoxyuridine for uridine. mRNA was capped co-transcriptionally using CleanCap AG analog (TriLink 
Biotechnologies) resulting in a 5' Cap 1 structure. In vitro transcription reaction was performed as previously described ${ }^{67}$ with the following changes; $16.5 \mathrm{mM}$ magnesium acetate and $4 \mathrm{mM}$ CleanCap AG were used as the final concentration during transcription, and mRNAs were purified using RNeasy kit (QIAgen). Mammalian-optimized UTR sequences (TriLink) and a 120-base polyA tail were included in the transcribed PCR product.

To edit Jurkat T cells, $1 \times 10^{6}$ were centrifuged at $500 \times g$ for 5 min, washed with $1 \mathrm{X}$ PBS, and centrifuged again at $500 \times g$ for $5 \mathrm{~min}$. The cells were resuspended in $12 \mathrm{uL}$ of plain RPMI 1640, $3 \mu \mathrm{g}$ of evoCDAmax and $100 \mu \mathrm{M}$ IDT-synthesized guide RNA was added, and cells were nucleofected using the Neon transfection system program $1600 \mathrm{~V}, 3$ pulses, 10ms. Cells were ejected into RPMI. rs72928038 base-edited cells were either left alone or combined with safe harbor base-edited cells (termed WT for the purposes of this study). The cells were incubated for 7 days prior to harvesting for PrimeFlow.

For PrimeFlow, 10 million cells were aliquoted in PBS in polypropylene tubes and centrifuged at $500 \times g$ for 5 min. All but $100 \mu \mathrm{L}$ of the supernatant was discarded (this step is true for every centrifugation step in this protocol) and the cells were resuspended in the residual volume. Cells were then fixed according to the manufacturer's protocol (ThermoFisher, 8818005-210) using Fixation Buffer 1 for 30 min rotating at $2-8^{\circ} \mathrm{C}$. Cells were then centrifuged at $800 \times g$ for 5 min and the supernatant was discarded. Cells were then permeabilized according to manufacturer's protocol with addition of RNase inhibitors through inversion, and centrifugation at $800 \times g$ for $5 \mathrm{~min}$, then the supernatant was discarded. This step was repeated. A second fixation step was carried out using Fixation Buffer 2 according to manufacturer's protocol, the samples were mixed, and inverted for $1 \mathrm{~h}$ in the dark at RT. The cells were then centrifuged at $800 \times g$ for 5 min at RT, and the samples were washed twice with PrimeFlow RNA Wash Buffer, 
centrifuging the samples at $800 \times g$ between each wash for $5 \mathrm{~min}$. The $B A C H 2$ target probe (ThermoFisher) was added at $1 \mathrm{X}$ in PrimeFlow RNA Target Probe Diluent, mixed thoroughly by pipetting up and down $\left(100 \mu \mathrm{L}\right.$ of probe/sample), and incubated at $40{ }^{\circ} \mathrm{C}$ for $2 \mathrm{~h}$, with inversion every 30 min. $1 \mathrm{~mL}$ of PrimeFlow RNA Wash Buffer was added to each sample, the samples were inverted to mix, and centrifuged at $800 \times g$ for $5 \mathrm{~min}$, and the supernatant was aspirated. Samples were then washed with $1 \mathrm{~mL}$ PrimeFlow RNA Wash Buffer containing RNase inhibitors twice, followed by centrifugation at $800 \times g$ for 5 min. $100 \mu \mathrm{L}$ of PrimeFlow RNA PreAmp Mix was then added to each sample and briefly vortexed to mix, and the samples were then incubated for $1.5 \mathrm{~h}$ at $40{ }^{\circ} \mathrm{C}$ with mild vortexing once every $30 \mathrm{~min}$. Samples were washed three times with $1 \mathrm{~mL}$ of PrimeFlow RNA Wash Buffer, and then were centrifuged at $800 \times g$ for $5 \mathrm{~min}$, and the supernatant was aspirated. $100 \mu \mathrm{L}$ of PrimeFlow RNA Amp Mix was then added to each sample, the samples were mixed by vortexing, and incubated for $1.5 \mathrm{~h}$ at $40{ }^{\circ} \mathrm{C}$ with mild vortexing once every $30 \mathrm{~min}$. The cells were then washed twice in $1 \mathrm{~mL}$ of PrimeFlow RNA Wash Buffer and centrifuged at $800 \times g$ for $5 \mathrm{~min}$. Each sample received $100 \mu \mathrm{L}$ of PrimeFlow RNA Label Probe diluted in PrimeFlow RNA Label Probe Diluent and incubated for $1 \mathrm{~h}$ at $40{ }^{\circ} \mathrm{C}$ with mild vortexing once at $30 \mathrm{~min}$. Samples were then washed with $1 \mathrm{~mL}$ of PrimeFlow RNA Wash Buffer at RT followed by centrifugation at $800 \times g$ for $5 \mathrm{~min}$. The samples were then washed five times with $35^{\circ} \mathrm{C}$ PrimeFlow RNA Wash Buffer following each wash with centrifugation at $800 \times g$ for $5 \mathrm{~min}$. Samples were then left in $100 \mu \mathrm{L}$ of PBS and stored in the dark at $4{ }^{\circ} \mathrm{C}$ until sorting.

Base edited $B A C H 2$ PrimeFlowed cells were cell sorted into six $10.5 \%$ bins, sorting on the extremes of expression $(30 \%$ on either the low or high portion of the expression distribution, each divided into three contiguous bins each comprising $\sim 10.5 \%$ of the overall distribution) 
using a Beckman Coulter MoFlo Astrios instrument. For each independent experiment, 100K cells were sorted per bin. Genomic DNA for each sample was then reverse-crosslinked using ChIP Lysis Buffer (1\% SDS, 0.01 M EDTA, 0.05 M Tris-HCl pH 7.5). Briefly, sorted cells were spun at $800 \times g$ for $10 \mathrm{~min}$ at $4^{\circ} \mathrm{C}$, the supernatant was aspirated, and the cells were resuspended in $50 \mu \mathrm{L}$ of ChIP Lysis Buffer, and incubated at $65^{\circ} \mathrm{C}$ for $10 \mathrm{~min}$. The samples were then cooled to $37^{\circ} \mathrm{C}$ and $2 \mu \mathrm{L}$ of RNase Cocktail (ThermoFisher, AM2286) was added to each sample and the sample was mixed well by pipetting, followed by incubation at $37^{\circ} \mathrm{C}$ for $30 \mathrm{~min} .10 \mu \mathrm{L}$ of Proteinase K (NEB, P8107S) was added to each sample and the sample was mixed well by pipetting, followed by incubation at $37^{\circ} \mathrm{C}$ for $2 \mathrm{~h}$ and then $95^{\circ} \mathrm{C}$ for $20 \mathrm{~min}$. gDNA was extracted using Agencourt XP beads at 0.7X following the manufacturer's protocol, and the sample was eluted at $100 \mu \mathrm{L}$. rs72928038 region amplicon libraries were prepared by nested PCR of each sample, splitting each into two $50 \mu \mathrm{L}$ reactions $(25 \mu \mathrm{L}$ NEBNext Master Mix, $2.5 \mu \mathrm{L} 10 \mu \mathrm{M}$ BACH2_gDNAamp_F and $2.510 \mu \mathrm{M}$ BACH2_gDNAamp_R, and $20 \mu \mathrm{L}$ of reverse-crosslinked sample), program: $98^{\circ} \mathrm{C}$ for $30 \mathrm{~s}, 23$ cycles of $98^{\circ} \mathrm{C}$ for $15 \mathrm{~s}, 65^{\circ} \mathrm{C}$ for $30 \mathrm{~s}$, $72{ }^{\circ} \mathrm{C}$ for $1 \mathrm{~min}$, then $72^{\circ} \mathrm{C}$ for $2 \mathrm{~min}$, followed by $1.5 \mathrm{X}$ Ampure XP cleanup, and elution in 25 $\mu \mathrm{L}$ EB. A second $50 \mu \mathrm{L}$ PCR was performed to add Nextera adapters (25 $\mu \mathrm{L}$ NEBNext Master Mix, $2.5 \mu \mathrm{L} 10 \mu \mathrm{M}$ BACH2_gseq_F and $2.510 \mu \mathrm{M}$ BACH2_gseq_R, and $20 \mu \mathrm{L}$ of Ampurecleaned PCR1), program: $98^{\circ} \mathrm{C}$ for $30 \mathrm{~s}, 6$ cycles of $98^{\circ} \mathrm{C}$ for $15 \mathrm{~s}, 64^{\circ} \mathrm{C}$ for $30 \mathrm{~s}, 72^{\circ} \mathrm{C}$ for $30 \mathrm{~s}$, then $72{ }^{\circ} \mathrm{C}$ for $2 \mathrm{~min}$, followed by $1.5 \mathrm{X}$ Ampure XP cleanup, and elution in $25 \mu \mathrm{L} \mathrm{EB}$. A final 50 $\mu \mathrm{L}$ PCR to add Nextera barcodes and sequencing adapters was then performed $(25 \mu \mathrm{L}$ NEBNext Master Mix, $2.5 \mu \mathrm{L}$ of mixed $10 \mu \mathrm{M}$ barcoded Nextera_F and Nextera_R, and 22.5 $\mu \mathrm{L}$ Ampurecleaned PCR2) for 6 cycles; program: $98^{\circ} \mathrm{C}$ for $30 \mathrm{~s}, 25$ cycles of $98^{\circ} \mathrm{C}$ for $15 \mathrm{~s}, 62^{\circ} \mathrm{C}$ for $15 \mathrm{~s}$, $72{ }^{\circ} \mathrm{C}$ for $16 \mathrm{~s}$, then $72^{\circ} \mathrm{C}$ for $2 \mathrm{~min}$. The libraries were then quantified by Qubit and 
TapeStation, mixed at equimolar ratios, PhiX (Illumina, FC-110-3001) was added at 20\%, and samples were sequenced aiming to get $>1,000,000$ reads per bin, on either an Illumina Nextseq 550 or MiSeq.

CRISPResso (version 2.0.29) ${ }^{68}$ was used to count the genotypes of each of the base editor-induced mutations present within the sequencing data associated with each FACS sorting bin. The read counts and genotypes for each sorting bin and the unsorted cells as output by CRIPSResso, were input into R, and MAUDE (version 0.99.3) ${ }^{69}$ was used to infer the expression levels of genotype, separately for each experiment. Here, we assumed that $10.5 \%$ of the cells were sorted into each of the sorting bins, which was the approximate number observed to fall into each bin during the experiments. We used MAUDE's 'findGuideHitsAllScreens' function to identify the mean expression associated with each genotype (treating genotypes as MAUDE "guides"), using default parameters. The statistical effect of rs72928038 base edits compared to WT on $B A C H 2$ expression were calculated using a paired (by experiment) one-sided Student's $t$ test with unequal variance.

\section{ATAC-seq}

We used the FAST-ATAC protocol ${ }^{11}$. Human primary T cells from female subjects (age 12-46) were isolated from blood by Ficoll, followed by flow sorting of live cell single lymphocytes, $\mathrm{CD}^{+} \mathrm{CD}^{+}$. Cells were sorted into RPMI with $10 \% \mathrm{FBS}$, and were immediately processed for ATAC-seq. 10,000-40,000 cells were sorted into RPMI 1640 containing 10\% fetal bovine serum. The cells were centrifuged at $500 \times g$ for 5 min at $4{ }^{\circ} \mathrm{C}$. All of the supernatant was aspirated, ensuring that the pellet was not disturbed in the process. The pellet was then resuspended in the tagmentation reaction mix ( $25 \mu \mathrm{L} 2 \mathrm{X}$ TD Buffer (Illumina, 15027866), $2.5 \mu \mathrm{L}$ TD Enzyme (Illumina, 15038061), $0.5 \mu \mathrm{L}$ 1\% Digitonin (Promega, G9441), $22 \mu \mathrm{L} \mathrm{H}_{2} \mathrm{O}$ ) and 
mixed at 300 RPMs at $37^{\circ} \mathrm{C}$ for 30 min on an Eppendorf Thermomixer. Immediately after the incubation, samples were purified using a minElute kit (Qiagen, 28006), eluting in $10 \mu \mathrm{L}$. The entire sample underwent PCR amplification (a $50 \mu \mathrm{L}$ reaction with $25 \mu \mathrm{L}$ NEBNext, $2.5 \mu \mathrm{L}$ of mixed Nextera_F and Nextera_R (10 $\mu \mathrm{M}$ each; Supplementary Table 14), $10 \mu \mathrm{L}$ of tagmented DNA, and $12.5 \mu \mathrm{L} \mathrm{H} 2 \mathrm{O})$ for five cycles with the following program $\left(72{ }^{\circ} \mathrm{C}, 5 \mathrm{~min} ; 98^{\circ} \mathrm{C}, 30 \mathrm{~s}\right.$; five cycles of $98^{\circ} \mathrm{C}, 15 \mathrm{~s}, 63^{\circ} \mathrm{C}, 15 \mathrm{~s}, 72^{\circ} \mathrm{C}, 1 \mathrm{~min}$ ). We performed qPCR with $5 \mu \mathrm{L}$ of the sample to determine the number of additional cycles required, while the rest of the sample remained on ice. The $5 \mu$ of sample was added to a qPCR mix $(5 \mu \mathrm{L}$ of PCR, $5 \mu$ of NEBNext, $0.5 \mu \mathrm{L}$ mixed Nexter_F and Nextera_R primers, $0.09 \mu \mathrm{L}$ of 100X SYBR (Invitrogen, S7563), $4.41 \mu \mathrm{L} \mathrm{H} 2 \mathrm{O})$ and $\mathrm{qPCRed}\left(98^{\circ} \mathrm{C}, 30 \mathrm{~s} ; 20\right.$ cycles of $\left.98^{\circ} \mathrm{C}, 15 \mathrm{~s}, 63{ }^{\circ} \mathrm{C}, 15 \mathrm{~s}, 72^{\circ} \mathrm{C}, 1 \mathrm{~min}\right)$. The number of cycles that it took to reach $1 / 3$ the maximum fluorescence threshold in the qPCR was then applied via PCR to the original PCR sample. Libraries were cleaned using 1.5X Agencourt XP beads and ethanol washes per manufacturer's protocol. The DNA concentration of the sample was measured using Qubit and the average fragment size was determined using a TapeStation. Samples were then multiplexed and sequenced using 50 bp paired end chemistry at an average read-count of $30 \mathrm{M}$ reads per sample.

Paired-end ATAC-seq reads were mapped to the genome (hg19) using Bowtie $2^{70}$ (v2.2.1; parameters: --maxins 2000), with duplicate reads removed using Picard (v2.20.6;

MarkDuplicates REMOVE_DUPLICATES=true), and peaks called using HOMER (v4.6; findPeaks -style dnase).

The comparison of the allelic bias of risk and non-risk alleles in accessible chromatin data from heterozygous individuals was conducted through the use of a binomial test for each sample. Samples indicated in red (Fig. 4C) had significant allelic bias. 


\section{Creation of Bach2 enhancer mutant and knockout mice}

All animal procedures were performed in accordance with The Jackson Laboratory, Broad Institute, and Benaroya Research Institute Institutional Animal Care and Use Committees.

The mouse syntenic region including rs72928038 and EH38E2485452 was determined using LiftOver (UCSC) and conservation with human sequence was calculated using EMBOSS Matcher ${ }^{58}$. Bach2 ${ }^{18 \mathrm{del}}$ mice were generated using direct delivery of CRISPR-Cas 9 reagents to mouse zygotes following the protocol of Qin et al. including guide design and electroporation ${ }^{59}$. The sequence of guide RNA IDT1038 and ssDO Donor10653 to create Bach2 ${ }^{18 \mathrm{del}}$ is listed in Supplementary Table 14. crRNA containing target sequence (Alt-R CRISPR-Cas9, IDT, 1072532) and tracRNA (Alt-R tracrRNA, IDT,1072534) were hybridized according to the manufacturer's protocol. The guideRNA duplex was mixed with AltR-SpCas9 V3 (IDT, 108105) in $\mathrm{TE}(\mathrm{pH} 7.5)$ and incubated for $20 \mathrm{~min}$ at room temperature followed by adding ssDO and centrifuging at 14,000 RPM in a microcentrifuge. The final concentrations of the gRNA, spCas9 and ssDO were $600 \mathrm{ng} / \mu 1,500 \mathrm{ng} / \mu \mathrm{l}$ and $1000 \mathrm{ng} / \mu \mathrm{l}$ respectively. $10 \mu \mathrm{l}$ of the supernatant of the mixture was mixed with $10 \mu \mathrm{l}$ of Opti-MEM (Thermo Fisher, 3198570) and zygotes treated with acidic Tyrode's solution (Millapore-Sigma, T1788) for $10 \mathrm{sec}$ and washed with pre-warmed M2 media (Millapore-Sigma, M7167). Electroporation was performed by using ECM830 Square Wave Electroporation System (BTX, 45-0661) with $1 \mathrm{~mm}$ electroporation cuvette (Harvard Apparatus, 45-0124) in the following conditions: $2 \times 1 \mathrm{~ms}$ pulses at $30 \mathrm{~V}$ with $100 \mathrm{~ms}$ interval. After recovery in $100 \mu \mathrm{l}$ of M2 media, embryos were transferred into B6Qsi5F1 pseudopregnant female mice.

Genotypes of founder mice were checked by Illumina sequencing. A 384 bp genomic fragment surrounding the target reference (mm10, chr4:32263658-32264041) was amplified 
from genomic DNA isolated from tail and peripheral blood using $1 \mu$ l of prepped DNA in $20 \mu 1$ of PCR reaction containing $0.4 \mu 1$ of PrimerStar GXL (TAKARA Bio, R050A), $4 \mu 1$ of 5× Buffer, $2 \mu 1$ of $10 \mathrm{mM}$ each dNTP mixture, $0.5 \mu 1$ each of Bach2ilmn_F1 and Bach2ilmn_R1 primers $\left(10 \mu \mathrm{M}\right.$, Supplementary Table 14) with the following conditions: $98{ }^{\circ} \mathrm{C}$ for $2 \mathrm{~min}, 20$ cycles of $\left(98^{\circ} \mathrm{C}\right.$ for $10 \mathrm{sec}, 62^{\circ} \mathrm{C}$ for $15 \mathrm{sec}, 68^{\circ} \mathrm{C}$ for $\left.60 \mathrm{sec}\right), 68^{\circ} \mathrm{C}$ for $2 \mathrm{~min}$. PCR products were purified by using $1.1 \times$ volume of AMPure XP and eluted by $20 \mu$ of EB. P5/P7 tags were added using $10 \mu \mathrm{l}$ of first PCR product in a $50 \mu \mathrm{l}$ of PCR reaction containing $25 \mu 1$ of Q5 NEBNext master mix, $2.5 \mu \mathrm{l}$ each of TruSeq multiplexing primers $(10 \mu \mathrm{M})$ with the following conditions: $98^{\circ} \mathrm{C}$ for $30 \mathrm{sec}, 6$ cycles of $\left(98^{\circ} \mathrm{C}\right.$ for $10 \mathrm{sec}, 62^{\circ} \mathrm{C}$ for $20 \mathrm{sec}, 72{ }^{\circ} \mathrm{C}$ for $\left.30 \mathrm{sec}\right), 72$ ${ }^{\circ} \mathrm{C}$ for 5 min. Amplified products were purified by using $1.2 \times$ volume of AMPure XP, eluted by $20 \mu 1$ of EB and pooled with the same amount. The mixed library was sequenced on MiSeq (Illumina) using $2 \times 250$ bp chemistry of nano v2 reagent.

\section{Isolation of primary mouse $\mathbf{T}$ cells}

Mouse primary naïve CD8 T cells were isolated from the spleens of WT or Bach $2^{18 \mathrm{del}}$ mice through sorting on live single lymphocytes, $\mathrm{CD} 3^{+} \mathrm{CD}^{+} \mathrm{CD} 62 \mathrm{~L}^{\text {hi }} \mathrm{CD} 44^{\text {lo }}$ into $\mathrm{PBS}$ containing $2 \%$ of FBS. Cells were spun at $500 \times g$ for 5 min and lysed by RLT buffer with $40 \mathrm{mM}$ DTT, followed by processing for BRB-seq.

\section{BRB-seq}

Naïve T cells were sorted from spleens collected from 21 -week-old females. 5 x $10^{5}$ cells for each replicate were sorted by using BD FACSymphony S6 with a $70 \mu \mathrm{m}$ nozzle. The fluorophore-conjugated antibodies and dilutions used for cell sorting are listed in Supplementary Table 14. Total RNA from sorted cells was isolated by using RNeasy plus micro (QIAGEN, 74034). $50 \mathrm{ng}$ for each sample was used for the reverse transcription with barcoded primer BU3 
(IDT; Supplementary Table 14) followed by the purification, second strand synthesis and tagmentation following the original BRB-seq protocol but using AMPure XP for purification ${ }^{34}$. Tagmented library was amplified with P5_BRB and BRB_Idx7N5 primers ( $5 \mu \mathrm{L}$, Supplementary Table 14) using NEBNext UltraTM II Q5 Master Mix (NEB, M0544L) which was incubated at $98^{\circ} \mathrm{C}$ for $30 \mathrm{sec}$ before adding DNA with the following conditions: $72{ }^{\circ} \mathrm{C} 3 \mathrm{~min}, 98^{\circ} \mathrm{C}$ for 30sec, and 15 cycles of $\left(98^{\circ} \mathrm{C}\right.$ for $10 \mathrm{sec}, 63^{\circ} \mathrm{C}$ for $\left.30 \mathrm{sec}, 72^{\circ} \mathrm{C} 60 \mathrm{sec}\right), 72{ }^{\circ} \mathrm{C}$ for $5 \mathrm{~min}$. Libraries were sequenced by NextSeq 550 High Output with 21 bp for read 1 and 72 bp for read 2 (Illumina). Sequenced reads were aligned using STAR (v2.7.6a, --outFilterMultimapNmax 1) $)^{71}$ followed by demultiplexing using BRB-seq Tools (v1.6) $)^{34}$.

\section{BRB-seq differential expression and gene set enrichment analysis}

Counted unique UMIs for genes were normalized using variance stabilizing transformation (vst) in DESeq2 (v1.26.0) using default parameters ${ }^{72}$. Differential expression of genes was calculated using DESeq2 with default parameters and genes were then sorted by their differential expression test statistic as input into Gene set enrichment analysis (GSEA).

Expression heatmaps of differentially expressed genes in the Bach $2^{18 \mathrm{del}}$ mouse compared with WT littermates in Fig. 5e are based on row and column-normalized gene expression. Expression heatmaps of genes from CD8 Tscms with Bach2 gRNA-knockdown vs. empty vector control cells in Supplementary Fig. 11c are similarly based on row and column-normalized gene expression. Comparisons of gene expression for sample genes (Fig. 5d and 5h) were performed by plotting expression levels of each gene from DESeq2 normalized counts from WT and Bach $2^{18 d e l}$ naïve CD8 T cells, followed by performing a Student's unpaired t-test. Differences in CD62L mean fluorescence intensity between WT and Bach2 ${ }^{18 d e l}$ naïve CD8 T cells (Fig. 5e) was performed using an unpaired Student's $t$-test. 
GSEA v4.1.0 $0^{73}$ was performed from the differential expression ranked gene list. Mouse genes were collapsed to their human orthologs. The GSEAPreranked tool was used with minimum gene set size of 15 and maximum of 250; otherwise, all default parameters were used. The ImmunoSigDB immunologic signatures database (v7.2) ${ }^{74}$ was used for the gene sets in addition to gene sets comprised of differentially expressed genes from the re-analysis of the Bach2-perturbed Tscm RNA-seq data (as described below).

\section{Bach2-perturbed Tscm RNA-seq analysis}

Bach2-gRNA perturbed $(\mathrm{n}=3)$ and cognate empty vector control $(\mathrm{n}=3)$ RNA-seq data from Tscms ${ }^{10}$ was downloaded from NCBI GEO (GSE152379). Transcript quantification from raw RNA-seq data was performed using Kallisto v0.46.0 $0^{75}$ against the reference Mus musculus transcriptome index based on GRCm38 as provided by the software developers. Quantification of transcripts was performed using parameters --single -1 200 -s 20. Differential expression of genes in Bach2-gRNA perturbed vs. empty vector was performed using DESeq2 as described

above. Gene sets were constructed from the differential expression results based on genes with a Benjamini-Hochberg adjusted $\mathrm{P}$ value $<0.05)$. One gene set was comprised of the top 200 genes with increased expression in the gRNA vs. empty vector, and the other gene set consisted of the top 200 genes with decreased expression in gRNA vs. empty vector.

\section{Data visualization and processing}

Data visualization, exploratory data analysis, and processing were performed using R v3.6.2.

\section{Materials Availability}

- The Bach218del (stock \#35028) mouse strain is available from the Jackson Laboratory (Bar Harbor, ME).

- Plasmids generated in this study will be deposited to Addgene upon publication. 


\section{Data and code availability}

Datasets supporting this manuscript are freely available upon reasonable request from the corresponding author, and will be published on NCBI GEO upon the manuscript's acceptance.

\section{Code availability}

Code supporting this manuscript is freely available upon reasonable request from the corresponding author, and will be published on GitHub upon the manuscript's acceptance.

\section{REFERENCES}

1. Farh, K. K.-H. et al. Genetic and epigenetic fine mapping of causal autoimmune disease variants. Nature 518, 337-343 (2015).

2. Maurano, M. T. et al. Systematic localization of common disease-associated variation in regulatory DNA. Science 337, 1190-1195 (2012).

3. Schaid, D. J., Chen, W. \& Larson, N. B. From genome-wide associations to candidate causal variants by statistical fine-mapping. Nat. Rev. Genet. 19, 491-504 (2018).

4. Meuleman, W. et al. Index and biological spectrum of human DNase I hypersensitive sites. Nature 584, 244-251 (2020).

5. Finucane, H. K. et al. Partitioning heritability by functional annotation using genome-wide association summary statistics. Nat. Genet. 47, 1228-1235 (2015).

6. Ray, J. P. et al. Prioritizing disease and trait causal variants at the TNFAIP3 locus using functional and genomic features. Nat. Commun. 11, 1237 (2020).

7. Fulco, C. P. et al. Activity-by-contact model of enhancer-promoter regulation from thousands of CRISPR perturbations. Nat. Genet. 51, 1664-1669 (2019). 
8. Roychoudhuri, R. et al. BACH2 regulates $\mathrm{CD} 8(+) \mathrm{T}$ cell differentiation by controlling access of AP-1 factors to enhancers. Nat. Immunol. 17, 851-860 (2016).

9. Roychoudhuri, R. et al. BACH2 represses effector programs to stabilize Treg-mediated immune homeostasis. Nature 498, 506-510 (2013).

10. Yao, C. et al. BACH2 enforces the transcriptional and epigenetic programs of stem-like CD8+ T cells. Nat. Immunol. 22, 370-380 (2021).

11. Corces, M. R. et al. Lineage-specific and single-cell chromatin accessibility charts human hematopoiesis and leukemia evolution. Nat. Genet. 48, 1193-1203 (2016).

12. Calderon, D. et al. Landscape of stimulation-responsive chromatin across diverse human immune cells. Nat. Genet. 51, 1494-1505 (2019).

13. Tewhey, R. et al. Direct Identification of Hundreds of Expression-Modulating Variants using a Multiplexed Reporter Assay. Cell 165, 1519-1529 (2016).

14. Ulirsch, J. C. et al. Systematic Functional Dissection of Common Genetic Variation Affecting Red Blood Cell Traits. Cell 165, 1530-1545 (2016).

15. Klein, J. C. et al. A systematic evaluation of the design and context dependencies of massively parallel reporter assays. Nat. Methods 17, 1083-1091 (2020).

16. Inoue, F. et al. A systematic comparison reveals substantial differences in chromosomal versus episomal encoding of enhancer activity. Genome Res. 27, 38-52 (2017).

17. Liu, J. Z. et al. Association analyses identify 38 susceptibility loci for inflammatory bowel disease and highlight shared genetic risk across populations. Nat. Genet. 47, 979-986 (2015).

18. Beecham, A. H. et al. Analysis of immune-related loci identifies 48 new susceptibility variants for multiple sclerosis. Nat. Genet. 45, 1353-1360 (2013). 
19. International Multiple Sclerosis Genetics Consortium. Multiple sclerosis genomic map implicates peripheral immune cells and microglia in susceptibility. Science 365, eaav7188 (2019).

20. Onengut-Gumuscu, S. et al. Fine mapping of type 1 diabetes susceptibility loci and evidence for colocalization of causal variants with lymphoid gene enhancers. Nat. Genet. 47, 381-386 (2015).

21. Tsoi, L. C. et al. Identification of 15 new psoriasis susceptibility loci highlights the role of innate immunity. Nat. Genet. 44, 1341-1348 (2012).

22. Okada, Y. et al. Genetics of rheumatoid arthritis contributes to biology and drug discovery. Nature 506, 376-381 (2014).

23. Javierre, B. M. et al. Lineage-Specific Genome Architecture Links Enhancers and Noncoding Disease Variants to Target Gene Promoters. Cell 167, 1369-1384.e19 (2016).

24. Chen, J. et al. TAGAP instructs Th17 differentiation by bridging Dectin activation to EPHB2 signaling in innate antifungal response. Nat. Commun. 11, 1913 (2020).

25. Tamehiro, N. et al. T-cell activation RhoGTPase-activating protein plays an important role in Th17-cell differentiation. Immunol. Cell Biol. 95, 729-735 (2017).

26. Duke-Cohan, J. S. et al. Regulation of thymocyte trafficking by Tagap, a GAP domain protein linked to human autoimmunity. Sci. Signal. 11, eaan8799 (2018).

27. Yan, J., Pandey, S. P., Barnes, B. J., Turner, J. R. \& Abraham, C. T Cell-Intrinsic IRF5 Regulates T Cell Signaling, Migration, and Differentiation and Promotes Intestinal Inflammation. Cell Rep. 31, 107820 (2020).

28. Daley, S. R. et al. Rasgrp1 mutation increases naive T-cell CD44 expression and drives mTOR-dependent accumulation of Helios ${ }^{+}$T cells and autoantibodies. Elife 2, e01020 
(2013).

29. Priatel, J. J., Teh, S.-J., Dower, N. A., Stone, J. C. \& Teh, H.-S. RasGRP1 transduces lowgrade TCR signals which are critical for T cell development, homeostasis, and differentiation. Immunity 17, 617-627 (2002).

30. Schmiedel, B. J. et al. Impact of Genetic Polymorphisms on Human Immune Cell Gene Expression. Cell 175, 1701-1715.e16 (2018).

31. Moore, J. E. et al. Expanded encyclopaedias of DNA elements in the human and mouse genomes. Nature 583, 699-710 (2020).

32. Thuronyi, B. W. et al. Continuous evolution of base editors with expanded target compatibility and improved activity. Nat. Biotechnol. 37, 1070-1079 (2019).

33. Oki, S. et al. ChIP-Atlas: a data-mining suite powered by full integration of public ChIP-seq data. EMBO Rep. 19, e46255 (2018).

34. Alpern, D. et al. BRB-seq: ultra-affordable high-throughput transcriptomics enabled by bulk RNA barcoding and sequencing. Genome Biol. 20, 71 (2019).

35. Kakaradov, B. et al. Early transcriptional and epigenetic regulation of CD8 T cell differentiation revealed by single-cell RNA sequencing. Nat. Immunol. 18, 422-432 (2017).

36. Gautam, S. et al. The transcription factor c-Myb regulates CD8+ T cell stemness and antitumor immunity. Nat. Immunol. 20, 337-349 (2019).

37. Willinger, T. et al. Human naive CD8 T cells down-regulate expression of the WNT pathway transcription factors lymphoid enhancer binding factor 1 and transcription factor 7 (T cell factor-1) following antigen encounter in vitro and in vivo. J. Immunol. 176, 14391446 (2006).

38. Yamada, T., Park, C. S., Mamonkin, M. \& Lacorazza, H. D. Transcription factor ELF4 
controls the proliferation and homing of CD8+ T cells via the Krüppel-like factors KLF4 and KLF2. Nat. Immunol. 10, 618-626 (2009).

39. Araki, K. et al. Translation is actively regulated during the differentiation of CD8+ effector T cells. Nat. Immunol. 18, 1046-1057 (2017).

40. Buckler, J. L., Liu, X. \& Turka, L. A. Regulation of T-cell responses by PTEN. Immunol. Rev. 224, 239-248 (2008).

41. Liu, Y.-C. The E3 ubiquitin ligase Itch in T cell activation, differentiation, and tolerance. Semin. Immunol. 19, 197-205 (2007).

42. Reilly, S. K. et al. HCR-FlowFISH: A flexible CRISPR screening method to identify cisregulatory elements and their target genes. bioRxiv 2020.05.11.078675 (2020) doi:10.1101/2020.05.11.078675.

43. Yan, J. et al. Systematic analysis of binding of transcription factors to noncoding variants. Nature (2021) doi:10.1038/s41586-021-03211-0.

44. Lee, M. N. et al. Common Genetic Variants Modulate Pathogen-Sensing Responses in Human Dendritic Cells. Science. 343, 1246980-1246980 (2014).

45. Ye, C. J. et al. Intersection of population variation and autoimmunity genetics in human T cell activation. Science 345, 1254665 (2014).

46. Fairfax, B. P. et al. Innate immune activity conditions the effect of regulatory variants upon monocyte gene expression. Science 343, 1246949 (2014).

47. Ramos-Rodríguez, M. et al. The impact of proinflammatory cytokines on the $\beta$-cell regulatory landscape provides insights into the genetics of type 1 diabetes. Nat. Genet. 51, 1588-1595 (2019).

48. Negron, A., Robinson, R. R., Stüve, O. \& Forsthuber, T. G. The role of B cells in multiple 
sclerosis: Current and future therapies. Cell. Immunol. 339, 10-23 (2019).

49. Sahlén, P. et al. Chromatin interactions in differentiating keratinocytes reveal novel atopic dermatitis- and psoriasis-associated genes. J. Allergy Clin. Immunol. (2020) doi:10.1016/j.jaci.2020.09.035.

50. Boyd, M. et al. Characterization of the enhancer and promoter landscape of inflammatory bowel disease from human colon biopsies. Nat. Commun. 9, 1661 (2018).

51. Soemedi, R. et al. Pathogenic variants that alter protein code often disrupt splicing. Nat. Genet. 49, 848-855 (2017).

52. Griesemer, D. et al. Genome-wide functional screen of 3'UTR variants uncovers causal variants for human disease and evolution. bioRxiv 2021.01.13.424697 (2021) doi:10.1101/2021.01.13.424697.

53. Joshi, N. S. et al. Inflammation Directs Memory Precursor and Short-Lived Effector CD8+ T Cell Fates via the Graded Expression of T-bet Transcription Factor. Immunity 27, 281295 (2007).

54. Herndler-Brandstetter, D. et al. KLRG1+ Effector CD8+ T Cells Lose KLRG1, Differentiate into All Memory T Cell Lineages, and Convey Enhanced Protective Immunity. Immunity 48, 716-729.e8 (2018).

55. Ghoussaini, M. et al. Open Targets Genetics: systematic identification of trait-associated genes using large-scale genetics and functional genomics. Nucleic Acids Res. 49, D1311D1320 (2021).

56. Buniello, A. et al. The NHGRI-EBI GWAS Catalog of published genome-wide association studies, targeted arrays and summary statistics 2019. Nucleic Acids Res. 47, D1005-D1012 (2019). 
57. Hollenhorst, P. C. et al. DNA specificity determinants associate with distinct transcription factor functions. PLoS Genet. 5, e1000778 (2009).

58. Madeira, F. et al. The EMBL-EBI search and sequence analysis tools APIs in 2019. Nucleic Acids Res. 47, W636-W641 (2019).

59. Qin, W. et al. Efficient CRISPR/Cas9-Mediated Genome Editing in Mice by Zygote Electroporation of Nuclease. Genetics 200, 423-430 (2015).

60. Chang, C. C. et al. Second-generation PLINK: rising to the challenge of larger and richer datasets. Gigascience 4, 7 (2015).

61. Quinlan, A. R. \& Hall, I. M. BEDTools: a flexible suite of utilities for comparing genomic features. Bioinformatics 26, 841-842 (2010).

62. Coetzee, S. G., Coetzee, G. A. \& Hazelett, D. J. motifbreakR: an R/Bioconductor package for predicting variant effects at transcription factor binding sites. Bioinformatics 31, 38473849 (2015).

63. Kulakovskiy, I. V. et al. HOCOMOCO: towards a complete collection of transcription factor binding models for human and mouse via large-scale ChIP-Seq analysis. Nucleic Acids Res. 46, D252-D259 (2018).

64. Heinz, S. et al. Simple combinations of lineage-determining transcription factors prime cisregulatory elements required for macrophage and B cell identities. Mol. Cell 38, 576-589 (2010).

65. Gate, R. E. et al. Genetic determinants of co-accessible chromatin regions in activated T cells across humans. Nat. Genet. 50, 1140-1150 (2018).

66. Lee, D. et al. A method to predict the impact of regulatory variants from DNA sequence. Nat. Genet. 47, 955-961 (2015). 
67. Vaidyanathan, S. et al. Uridine Depletion and Chemical Modification Increase Cas9 mRNA Activity and Reduce Immunogenicity without HPLC Purification. Mol. Ther. Nucleic Acids 12, 530-542 (2018).

68. Pinello, L. et al. Analyzing CRISPR genome-editing experiments with CRISPResso. Nat. Biotechnol. 34, 695-697 (2016).

69. de Boer, C. G., Ray, J. P., Hacohen, N. \& Regev, A. MAUDE: inferring expression changes in sorting-based CRISPR screens. Genome Biol. 21, 134 (2020).

70. Langmead, B. \& Salzberg, S. L. Fast gapped-read alignment with Bowtie 2. Nat. Methods 9, 357-359 (2012).

71. Dobin, A. et al. STAR: ultrafast universal RNA-seq aligner. Bioinformatics 29, 15-21 (2013).

72. Love, M. I., Huber, W. \& Anders, S. Moderated estimation of fold change and dispersion for RNA-seq data with DESeq2. Genome Biol. 15, 550 (2014).

73. Subramanian, A. et al. Gene set enrichment analysis: A knowledge-based approach for interpreting genome-wide expression profiles. Proc. Natl. Acad. Sci. U S A. 102, 1554515550 (2005).

74. Godec, J. et al. Compendium of Immune Signatures Identifies Conserved and SpeciesSpecific Biology in Response to Inflammation. Immunity 44, 194-206 (2016).

75. Bray, N. L., Pimentel, H., Melsted, P. \& Pachter, L. Near-optimal probabilistic RNA-seq quantification. Nat. Biotechnol. 34, 525-527 (2016).

76. Kent, W. J., Zweig, A. S., Barber, G., Hinrichs, A. S. \& Karolchik, D. BigWig and BigBed: enabling browsing of large distributed datasets. Bioinformatics 26, 2204-2207 (2010).

77. Ramírez, F. et al. deepTools2: a next generation web server for deep-sequencing data 
analysis. Nucleic Acids Res. 44, W160-5 (2016).

\section{ACKNOWLEDGMENTS}

We gratefully acknowledge the contribution of Richard Maser and Genetic Engineering Technologies Service, Jeniffer Kelmenson and Transgenic Genotyping Service, William Schott and Flow Cytometry Service, and Ryan Lynch and Genome Technologies Service at The Jackson Laboratory, Anton McCaffrey and Jordana Henderson at Trilink Biotechnologies, the Broad Institute vivarium, Flow Cytometry Core, and Genomics Core, and the Benaroya Research Institute vivarium and Flow Cytometry Core for expert assistance with the work described in this manuscript. We thank Dr. Peter Gregersen and Dr. Betty Diamond for providing genotyped human PBMCs, and Dr. Susan Malkiel for help with processing human PBMCs for ATAC-seq and for review of the manuscript. We thank Dr. Virginia M. Green for her critical review of the manuscript. This work is funded by U.S. NIH K22 AI153648-01 (J.P.R.), R25 NS065745

(M.H.G.), U01 AI142756 (D.R.L.), RM1 HG009490 (D.R.L.), and R00 HG008179 (R.T.). G.A.N. acknowledges a Helen Hay Whitney postdoctoral fellowship. M.G. is the recipient of an EMBO Long-Term Fellowship (ALTF 486-2018) and a Cancer Research Institute/Bristol-Myers Squibb Fellow (CRI2993).

\section{AUTHOR CONTRIBUTIONS}

J.P.R., R.T., K.M., and M.H.G. conceived the study. J.P.R. performed MPRA, ATAC-seq on human CD4 T cells, base editing experiments, and luciferase, with the help of M.G. K.M. created the Bach2 ${ }^{18 \mathrm{del}}$ mouse line and performed RNA-seq on mouse naïve CD8 T cells. M.H.G, C.G.B., K.M., R.T., J.P.R. performed data analysis. G.A.N. and D.R.L. provided essential base editing 
reagents and critical advice for base editing experimental design. J.P.R. and M.H.G. wrote the manuscript with the help of K.M., R.T., and N.H. All authors have read and approved the manuscript.

\section{COMPETING INTERESTS}

G.A.N. and D.R.L have filed patent applications on genome editing agents. D.R.L. is a consultant and equity owner of Beam Therapeutics, Prime Medicine, and Pairwise Plants, companies that use genome editing. NH holds equity in BioNTech and consults for Related Sciences. Other authors have no conflicts of interest. 


\section{FIGURES AND LEGENDS}

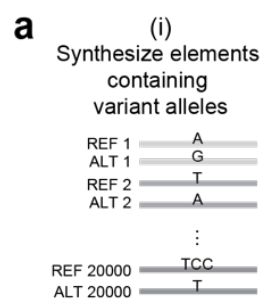

(iii)

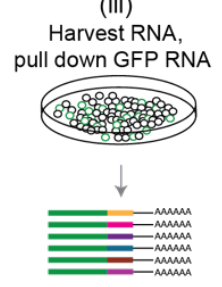

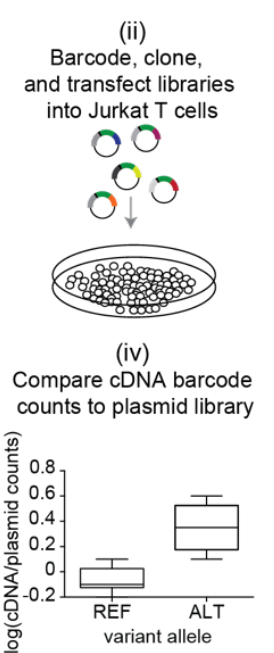

b

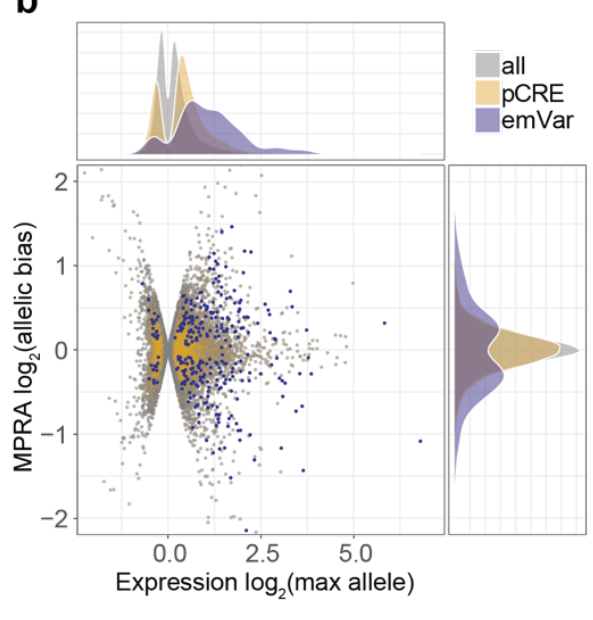

C Enrichment for fine-mapped variants

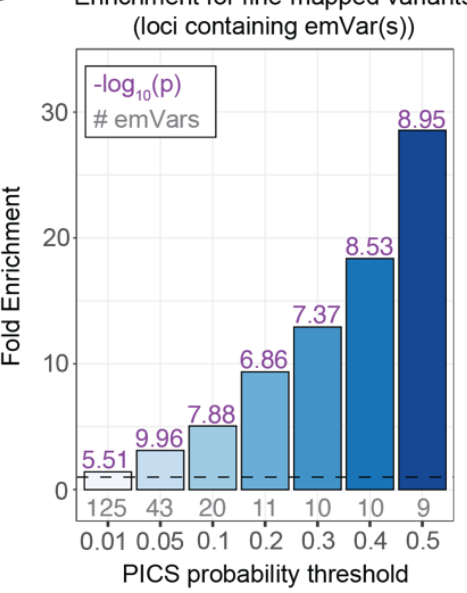

Fig. 1. Prioritizing GWAS variants using high-throughput reporter assays in Jurkat T

cells. a) Workflow for creating MPRA libraries- i) Oligonucleotide synthesis of variants and 200 bp surrounding genomic region; ii) barcoding, cloning, and transfection of plasmid library into Jurkat T cells; iii) harvesting RNA from Jurkat and pull down of GFP mRNA; iv) RNAsequencing of barcodes, normalization to their prevalence in the plasmid library, and comparison of alleles for differential reporter activity (a more detailed workflow is provided in Supplementary Fig. 1a). b) Volcano plot. The $\log _{2}$ expression value of the highest expressing allele is on the $\mathrm{X}$ axis, and the $\log _{2}$ of the activity of allele1/allele 2 is on the $\mathrm{Y}$ axis. $\mathrm{pCRE}=$ putative cis-regulatory element; emVar = expression-modulating variant. c) Bar plot showing enrichment of emVars for PICS statistically fine-mapped variants at GWAS loci where an emVar was detected, with the minimum PICS probability threshold indicated on the X axis. Gray numbers below each bar show the number of emVars that are statistically fine-mapped at a given PICS probability threshold. Purple numbers above each bar show the $-\log _{10}$ of the enrichment $\mathrm{P}$ value. Details of PICS enrichment results are shown in Table S9. Enrichment in (c) was 
bioRxiv preprint doi: https://doi.org/10.1101/2021.05.30.445673; this version posted May 30, 2021. The copyright holder for this preprint (which was not certified by peer review) is the author/funder. All rights reserved. No reuse allowed without permission.

calculated as a risk ratio (see Methods), and $\mathrm{P}$ values were determined through a two-sided Fisher's exact test. 
Figure 2

Enrichment for active elements in DHS

\section{a}

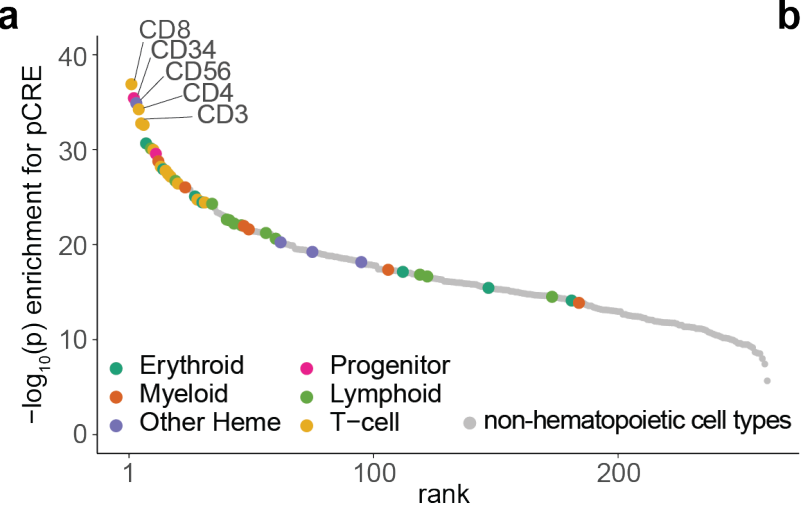

C

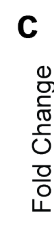

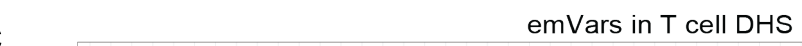

Enrichment for fine-mapped variants in DHS (loci containing emVar(s))

b

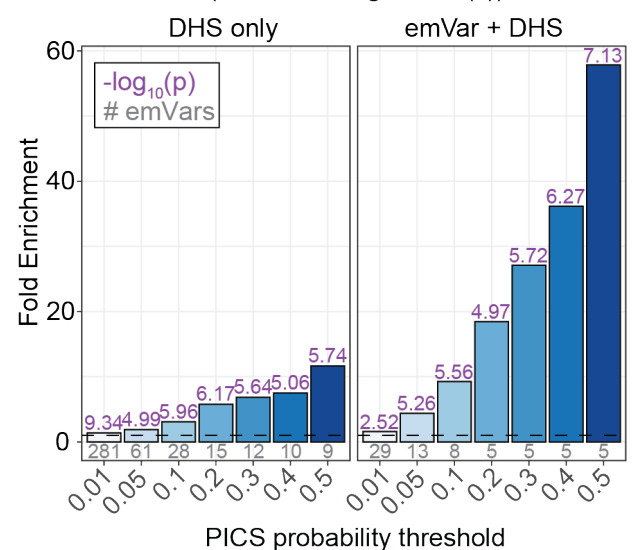

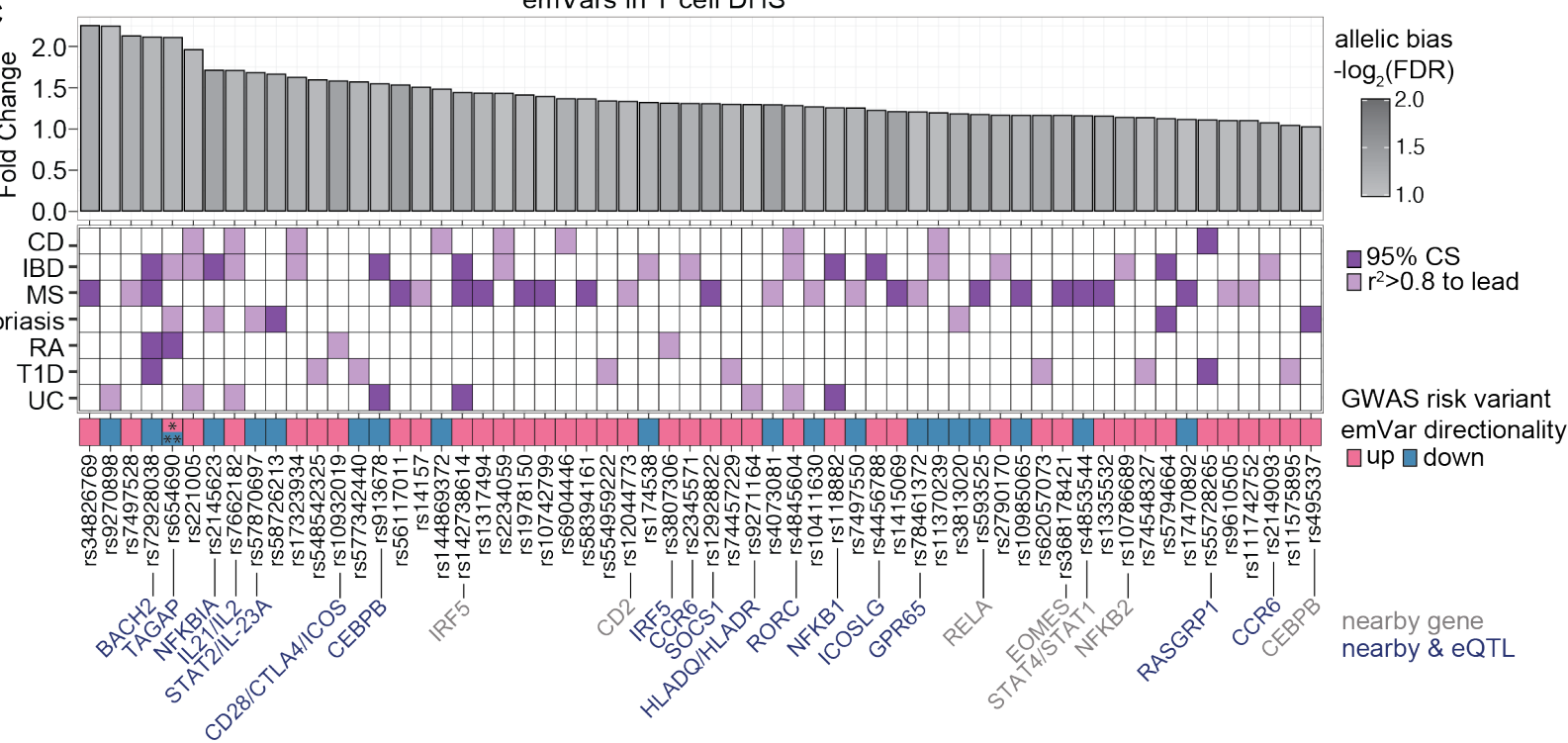

Fig. 2. T-GWAS emVars in $\mathrm{T}$ cell accessible chromatin enrich highly for fine-mapped

variants. a) Enrichment of DHS sites from hematopoietic and non-hematopoietic cell types for MPRA pCREs. Cell types are ranked from left to right from most statistically significant to least significant. Hematopoietic cell types are colored by their ontogeny as indicated in the legend. Non-hematopoietic cell types are shown in gray. Y-axis shows the $-\log _{10}$ of the enrichment P value. b) Enrichment of statistically fine-mapped variants within T cell DHS sites (left) and enrichment of statistically fine-mapped variants that are emVars within T cell DHS sites (right). 
Details of PICS enrichment results are shown in Table S9. c) Bar plot (top) of 60 emVars in T cell DHS sites with their allelic bias (y-axis) and $\log _{2}$ FDR (shade of bar). GWAS for which emVar is associated (middle). emVars in 95\% fine-mapping credible sets are shown in dark purple, while variants in tight LD to lead the variant $\left(\mathrm{r}^{2}>0.8\right)$ but not in credible sets are shown in light purple. Immediately underneath, pink and teal boxes indicate the MPRA expression directionality of the GWAS disease risk-increasing variant as compared to the non-risk variant, followed by variant rsIDs. For one variant, rs654690, the risk alleles are opposing depending on disease, with * indicating the risk allele for both psoriasis and IBD, and ** indicating the risk allele for RA. Nearby genes that are known to play a role in $\mathrm{T}$ cell differentiation and function (gray) and nearby genes for which the variant is an eQTL (dark blue; according to Open Targets Genetics; ${ }^{55}$ are listed on bottom. Enrichment (a) were determined through a two-sided Fisher's exact test. Enrichment in (b) was calculated as a risk ratio (see Methods), and P values were determined through a two-sided Fisher's exact test. Statistical significance of allelic bias in (c, top bar plot) was calculated using a paired Student's two-sided $t$-test. 
Figure 3

a

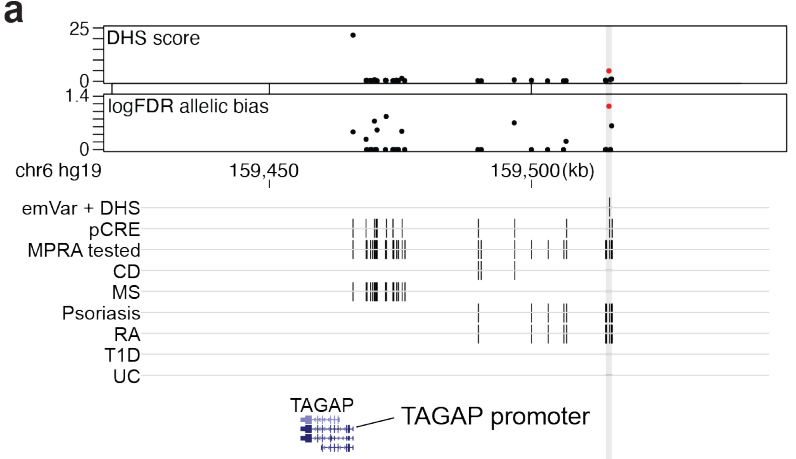

b

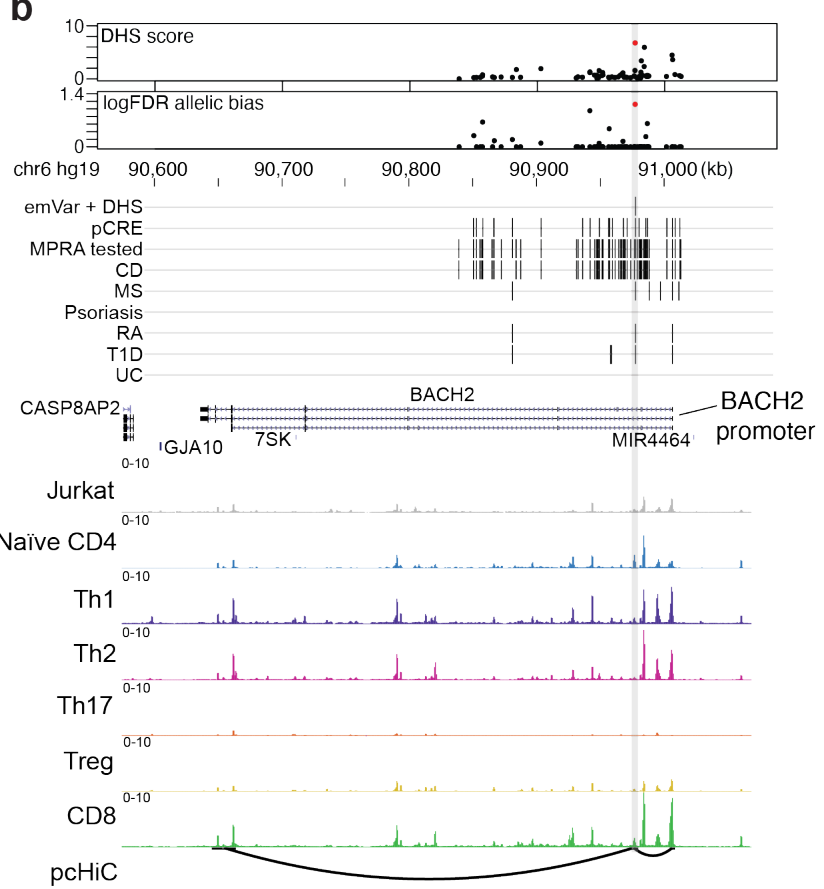

Fig. 3. Putative causal variants in a $B A C H 2$ intron and upstream of $T A G A P$. a and $b$ )

Dotplot (top) showing DHS signal (DHS score) and statistical significance of allelic bias $\left(\log _{10} \mathrm{FDR}\right.$ of MPRA allelic bias) for MPRA variants in the region; all tested variants on haplotype (black), significant emVars in DHS (red dot). Position of variants that are emVars, pCREs, variants tested in MPRA, and disease-associated variants for CD, MS, psoriasis, RA, T1D, and UC from the GWAS Catalog ${ }^{56}$ (middle). Genes in the locus are shown along with chromatin accessibility profiles (from Jurkat and specific $\mathrm{T}$ cell subsets) and $\mathrm{T}$ cell promoter capture $\mathrm{HiC}\left(\mathrm{pcHiC}^{23}\right)$ loops anchored on the region containing the emVar. $\mathrm{pcHiC}$ loops in (a) are specific to naïve $\mathrm{T}$ cells; $\mathrm{pcHiC}$ loop in (b) is present in all $\mathrm{T}$ cell subsets and conditions tested. Gray line depicts position of the prioritized emVar with respect to all data types. Statistical significance of allelic biases in (a) and (b) were calculated using a paired Student's two-sided $t$-test as described in Methods. 

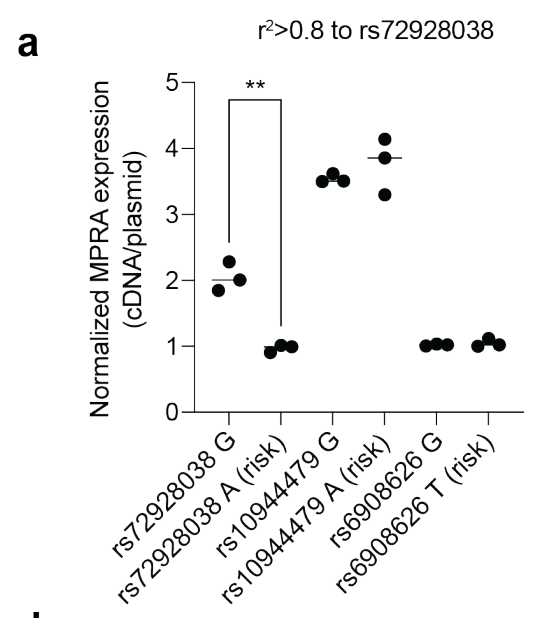

d

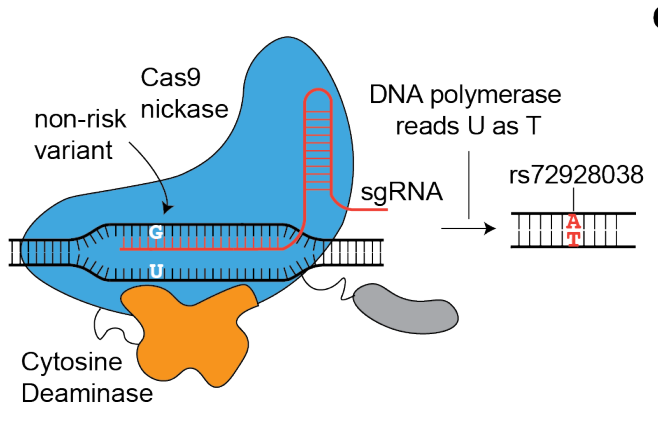

b
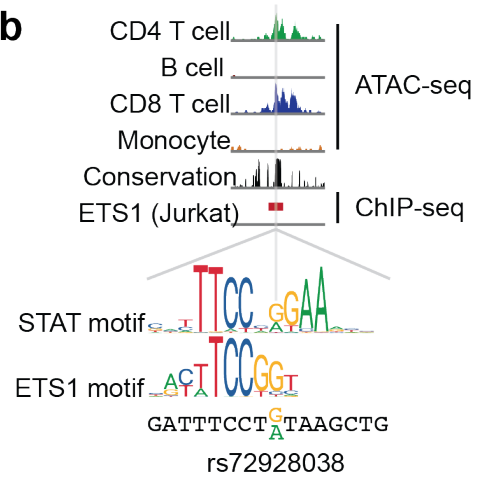

C

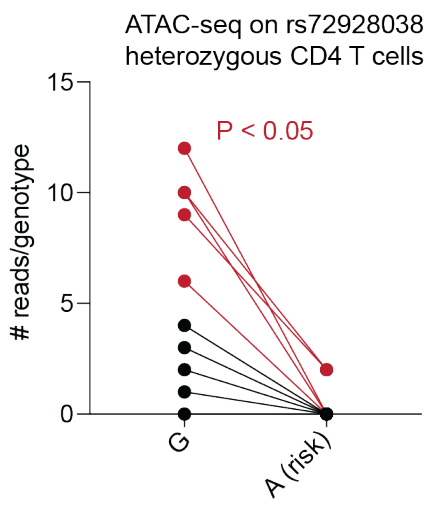

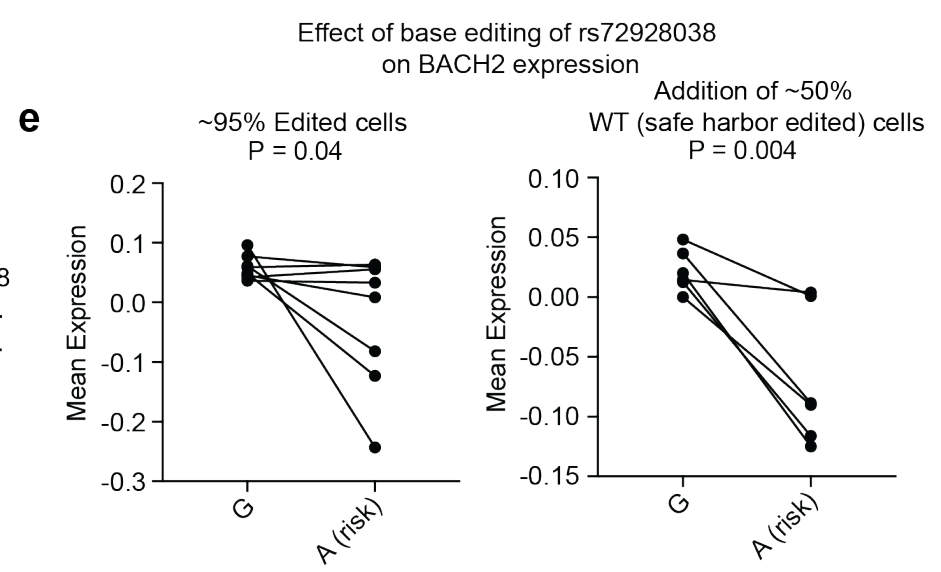

Fig. 4. Base editing of the $B A C H 2$ emVar (rs72928038) reduces $B A C H 2$ expression. a)

MPRA reporter expression of credible set variant alleles at the $B A C H 2$ locus $(\mathrm{n}=3$ independent

replicates). b) ATAC-seq profiles in CD4 T cells, B cells, CD8 T cells, and monocytes, vertebrate conservation, and ETS1 ChIP-seq ${ }^{57}$ at the site of rs72928038 (top). STAT and ETS1

TF motifs at the site of rs72928038 (bottom). c) ATAC-seq reads overlapping rs72928038 in CD4 T cells from heterozygous healthy individuals (10 genotyped individuals);. 5 of the 10 individuals (marked red) had a significant difference (at $P<0.05$ ) in number of reads between reference and alternate alleles according to a binomial test. d) Schematic of base editing rs 72928038 using the evoCDAmax cytosine base editor. e) PrimeFlow mean expression of $B A C H 2$ in cells containing the rs72928038 non-risk $(\mathrm{G})$ and base-edited risk (A) allele with rs 72928038 base-edited cells alone (left; 8 independent replicates) and when combined with cells 
that were edited at a safe harbor locus (right; 6 independent replicates). For (a), central tendency is shown as median and all points are plotted to show dispersion. For (e), central tendency is shown as mean and all points are plotted to show dispersion. P values determined by Student's two-sided t-test (a); Binomial test (c); Student's one-sided t-test (e). 
Figure 5

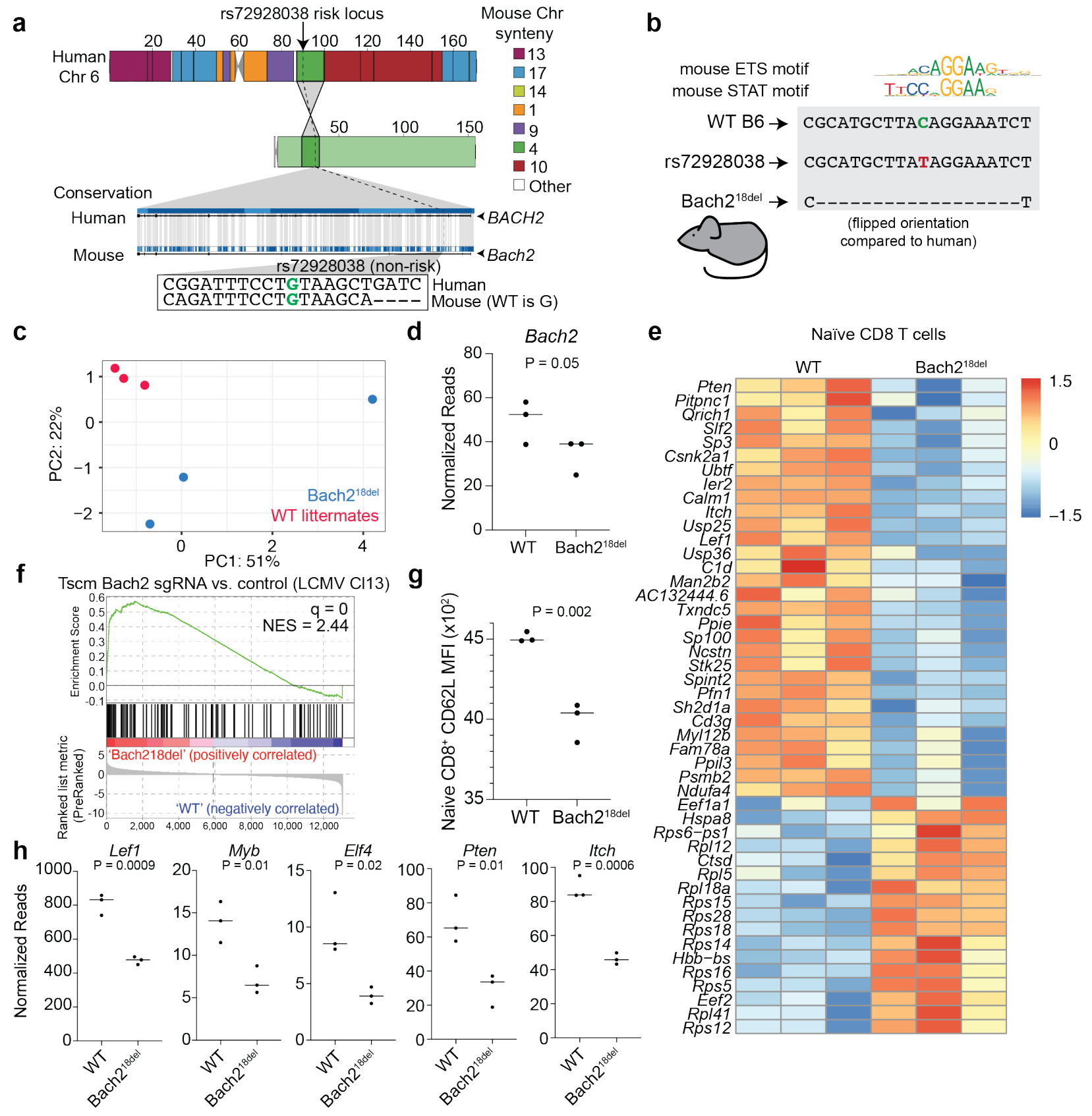

Fig. 5. Naïve T cells from mice with a deletion overlapping orthologous rs72928038 have

reduced transcriptional features of stemness. a) Synteny analysis of rs72928038 between

human and mouse. Location of rs72928038 (arrow and dotted line) on human chromosome 6 and mouse chromosome 4 (top) with colors indicating mouse chromosome synteny (see key).

Conservation of human $B A C H 2$ with mouse Bach2, with the location of rs 729280378 noted 
(dotted line), with inset showing conserved sequence between human and mouse at the site of rs 72928038 (bottom). b) Schematic of Bach2 ${ }^{18 \mathrm{del}}$ mutation. c) Principal components 1 and 2 from gene expression analysis of naïve CD8 T cells from Bach2 ${ }^{18 \mathrm{del}}$ and their WT littermates. d) Bach2 gene expression within naïve CD8 T cells from WT and Bach2 ${ }^{18 \mathrm{del}}$ mice. e) Expression heatmap of differentially expressed genes (adjusted $\mathrm{P}$ value $\leq 0.05$; calculated as described in Methods) between WT and Bach2 ${ }^{18 \text { del }}$ naïve CD8 T cells $(n=3$ per genotype). f) GSEA showing enrichment of Bach2 ${ }^{18 \mathrm{del}}$ vs. WT naïve CD8 T cells for a gene set derived from genes differentially expressed in Bach2 guide RNA-targeted CD8 Tscm cells vs. empty vector Tscm cells. Full GSEA results are shown in Table S13. g) Mean fluorescence intensity of CD62L surface expression on naïve WT and Bach $2^{18 d e l}$ CD8 T cells. h) Sample genes differentially expressed between WT and Bach $2^{18 d e l}$ mice in naïve CD8 T cells (c-h, $\mathrm{n}=3$ per genotype). $\mathrm{P}$ values determined by Student's one-sided t-test (d); Student's two-sided t-test (g and h). For (d), (g), and (h), central tendency shown as median and all points are plotted to show dispersion. Normalized enrichment score (NES) in (f) was calculated based on observed enrichment as compared to enrichments from permuted data as previously described and statistical significance shown as the false discovery rate $(\mathrm{q})^{58}$. 